\title{
Interplay between extreme drift and selection intensities favors the fixation of beneficial mutations in selfing maize populations
}

\author{
Arnaud Desbiez-Piat*, Arnaud Le Rouzic ${ }^{\dagger}$, Maud I. Tenaillon*,1 and Christine Dillmann*,1 \\ *Université Paris-Saclay, INRAE, CNRS, AgroParisTech, GQE - Le Moulon, France, ${ }^{\dagger}$ Université Paris-Saclay, CNRS, IRD, UMR Évolution, Génomes, \\ Comportement et Écologie, France.
}

\begin{abstract}
Population and quantitative genetic models provide useful approximations to predict long-term selection responses sustaining phenotypic shifts, and underlying multilocus adaptive dynamics. Valid across a broad range of parameters, their use for understanding the adaptive dynamics of small selfing populations undergoing strong selection intensity (thereafter High Drift-High selection regime, HDHS) remains to be explored. Saclay Divergent Selection Experiments (DSEs) on maize flowering time provide an interesting example of populations evolving under HDHS, with significant selection responses over 20 generations in two directions. We combined experimental data from Saclay DSEs, forward individual-based simulations, and theoretical predictions to dissect the evolutionary mechanisms at play in the observed selection responses. We asked two main questions: How do mutations arise, spread, and reach fixation in populations evolving under HDHS ? How does the interplay between drift and selection influence observed phenotypic shifts ? We showed that the long-lasting response to selection in small populations is due to the rapid fixation of mutations occurring during the generations of selection. Among fixed mutations, we also found a clear signal of enrichment for beneficial mutations revealing a limited cost of selection. Both environmental stochasticity and variation in selection coefficients likely contributed to exacerbate mutational effects, thereby facilitating selection grasp and fixation of small-effect mutations. Together our results highlight that despite a small number of polymorphic loci expected under HDHS, adaptive variation is continuously fueled by a vast mutational target. We discuss our results in the context of breeding and long-term survival of small selfing populations.
\end{abstract}

KEYWORDS Truncation selection, Experimental evolution, Adaptive dynamics, Distribution of fitness effects, Selection cost, Effective population size, Environmental stochasticity

Tnderstanding the evolutionary processes sustaining phenotypic shifts is at the core of quantitative genetic models. Empirical description of such shifts takes its roots in the breeding literature where truncation selection generates significant and sustainable responses (Hill and Caballero 1992; Walsh and Lynch 2018). Truncation selection is known to be the most effective form of directional selection (Crow and Kimura 1979). Under truncation selection, limits to the evolution of phenotypes are rarely reached as heritable variation persists through time (Odhiambo and Compton 1987; Moose et al. 2004; Weber and Diggins 1990; Caballero et al. 1991; Mackay 2010; Lillie et al. 2019). Such observations fit well with the breeder equation and its derivatives (Lush 1943; Lande 1979; Lande and Arnold 1983) which accurately predict selection response after one generation. With

${ }^{1}$ Corresponding authors: Université Paris-Saclay, INRAE, CNRS, AgroParisTech, GQE Le Moulon, 91190, Gif-sur-Yvette, France the additional hypothesis of constant genetic variance provided by the Fisher's infinitesimal model (Fisher 1930), theoretical models predict a continuous and linear response with no finite limits. However, the rate of response is expected to decline with selection-induced linkage disequilibrium (Bulmer 1971; Hospital and Chevalet 1996). Furthermore under finite population size, selection response is predicted to reach an asymptotic finite limit (Robertson 1960) as exemplified in mice (Roberts 1967; Falconer 1971). Results from other species are more equivocal (e.g. drosophila (Weber 1990; Weber and Diggins 1990; Weber 1996), or maize (Odhiambo and Compton 1987; Moose et al. 2004; Dudley and Lambert 2010; De Leon and Coors 2002; Lamkey 1992). Incorporation of de novo mutations indeed predicts a slower rate of response instead of a hard limit (Hill 1982b,a; Weber and Dig- 
gins 1990; Wei et al. 1996; Walsh and Lynch 2018). A sub-optimal average selection response is expected in two situations: when population size, $N$ is below $10^{4}$ reducing the genetic variance $\left(\widehat{V_{G}}\right)$ at mutation-drift equilibrium(Hill 1982b; Houle 1989); and when $\widehat{V_{G}}$ is reduced due to strong selection (Houle 1989). Overall, quantitative genetic models that include selection, drift and mutation (Houle 1989) are well-suited for predicting observed selection responses in a broad range of parameters (Hill and Rasbash 1986) - providing appropriate corrections, e.g. deviations from low drift and low selection intensity (Walsh and Lynch 2018). Most of these models, however, make the assumptions of random mating and of a probability of fixation of new mutations - determined by the product of population size by their selection coefficient, $N s$ - to be either $\ll 1$ or $\gg 1$. Mathematical models for the intermediate regime $N s \approx 1$ and non-random mating still remain unsatisfactory. Furthermore, the description of mechanisms of long-term selection response - and whether it can be understood and predicted by existing equations - has yet to be explored for polygenic traits evolving in small selfing populations under high selection intensity, a regime subsequently called HDHS (High-Drift High-Selection).

Both the Distribution of mutational Fitness Effects (DFE) and the mutation rate are central to long-term predictions of selection responses. Selection makes the DFE of fixed mutations different from that of incoming de novo mutations (Kassen and Bataillon 2006). In large populations, a high proportion of incoming de novo beneficial mutations are predicted to reach fixation, together with vanishing small effect deleterious mutations (Crow and Kimura 1971; Kimura 1983). In small populations and/or at small selection intensity, frequent loss of beneficial mutations due to drift together with the fixation of moderately strong deleterious mutations is expected. Hence Kimura's equation that links the fixation probability $\left(P_{f i x}\right)$ of a mutation to its frequency $(p)$, the population size $(N)$ and selective coefficient (s) $-P_{f i x}(s, p, N)=\left(1-e^{-4 s p N}\right) /\left(1-e^{-4 s N}\right)-$ applies to a vast range of parameters including $s$ values as high as 0.1 and $N$ as small as 10 individuals (Carr and Nassar 1970). An additional layer of complexity to DFE prediction comes from the mating system. Adaptation of very large asexual populations (such as microbes) is indeed affected by competition between alternative beneficial mutations occurring in different genetic background, a process referred to as clonal interference (Gerrish and Lenski 1998). Here the absence of recombination favors enrichment of the DFE in large beneficial mutational effects (Gerrish and Lenski 1998). However, if selection overpowers drift, i.e. Ns $\gtrsim 1$, or if the rate of beneficial mutation $\left(\mu_{B}\right)$ is small enough, the expected time lag between two successive mutations is sufficiently large for the first beneficial mutation to fix without interference of the second. While such behavior is expected when $N \mu_{B} \ll 1 / \ln (N s)$, for $N \mu_{B} \gtrsim 1 / \ln (N s)$ beneficial mutations evolve under clonal interference (Desai and Fisher 2007). Altogether these results highlight how the interplay of key parameters - $N, s, \mu$, effective recombination - determine the DFE and in turn, the long-term selection response.

Genomic footprints of selection have considerably enriched our vision of allele trajectories sustaining selection responses. On the one hand, one can observe genomic footprints such as hard and/or soft selective sweeps. A hard sweep is characterized by a strong decrease in genomic diversity at the selected locus and its surrounding region through genetic hitchhiking (Hermisson and Pennings 2017); while a soft sweep is associated with a weak genomic signature either because recombination on standing variation occurs so that a given advantageous mutation is associated with multiple haplotypes, or because recurrent de novo mutations are associated with multiple haplotypes. Together these footprints indicate that adaptation proceeds through a succession of sweeps at loci encoding the trait. On the other hand, absence of selection footprints is expected under the socalled polygenic selection model (Berg and Coop 2014; Wellenreuther and Hansson 2016; Walsh and Lynch 2018), that rather posits a collective response at many loci translating into simultaneous subtle shifts in allele frequencies, in compliance with the infinitesimal model. Whether adaptation proceeds through hard/soft sweeps or polygenic model primarily depends on the population-scaled mutation rate $(\theta)$ as well as the number of redundant loci that offer alternative ways for adaptation $(L)$ - the mutational target. Adaptation proceeds through hard sweeps for small $\theta \times L(\leq 0.1)$ while polygenic adaptation requires large $\theta \times L(\geq 100)$ with partial/soft sweeps in between (Messer and Petrov 2013; Höllinger et al. 2019). Extension of the hitchhiking model to a locus affecting a quantitative trait with an infinitesimal genetic background predicts that, under the hypothesis of a Gaussian fitness function, the fixation of a favorable mutation critically depends on the initial mutation frequency and the distance to the optimum (Chevin and Hospital 2008). Interestingly, while demographic parameters- population size, bottleneck strength - play a relatively small role in the speed of adaptation compared to standing and mutational variance, they change its qualitative outcome. Population bottlenecks diminish the number of segregating beneficial alleles, favoring hard sweeps from de novo mutations over soft sweeps from standing variation (Stetter et al. 2018).

By exploring short-term temporal dynamics of adaptation, experimental evolution has provided further hints into allele frequency changes, and into the extent of polymorphism and competition among beneficial mutations under various $\mathrm{drift} /$ selection/recombination regimes. Temporal dynamics are obtained either through pedigree information or time series samples. This last approach, widely used in microorganisms has revealed complex patterns of mutation spreading during the course of adaptation. These include clonal interference, the reduction of the relative advantage of a beneficial mutation in fit versus less fit genotypes (diminishing-return epistasis), and evidence for the same favorable mutation being selected in multiple independent evolved clones (genetic parallelism) (Good et al. 2017; Spor et al. 2014; Neher 2013; Good et al. 2012; Desai and Fisher 2007; Gerrish and Lenski 1998). However, in asexually reproducing microbes, adaptation proceeds through de novo mutations, which may reveal specific patterns not found in sexually-reproducing eukaryotes. In yeast, for instance, most adaptive changes correspond to the fixation of initial standing variation (Burke et al. 2014; Burke 2012). Patterns of allele frequency changes depend crucially on both $N_{e}$ and the frequency of sex, that are themselves intimately linked (see Hartfield et al. (2017)). Considering a single locus, fixation time decreases correlatively with the level of self-fertilization (Haldane 1927). At the same time, multilocus simulations have shown that selfing reduces effective population size through background selection and in turn, beneficial mutations are less likely to fix (Kamran-Disfani and Agrawal 2014; Roze 2016). In addition, as selection interference reduces the efficiency of selection in low-recombining regions, high selfing rates also increase the fixation of deleterious mutations through genetic hitchhiking (Hartfield and Glémin 2014). These insights are together in line 
with the low selection approximation that posits that reduction in effective recombination decreases selection efficiency.

In the current paper, we aimed at investigating the dynamics of the response to selection in small selfing populations evolving under high selection intensity. Situated at the parameters boundaries of current models, this regime is of particular interest to understand the limits of adaptation and long-term survival of small selfing populations undergoing strong selection. We relied here on two Divergent Selection Experiment (DSEs) conducted for 18 generations on Saclay's plateau (Saclay DSEs), south of Paris (France). These Saclay DSEs are ideal settings to address those issues: selection-by-truncation has been applied in a higher organism (maize), on a highly polygenic and integrated trait (flowering time, (Buckler et al. 2009; Tenaillon et al. 2018)) that directly affects fitness. Previous results indicate continuous phenotypic responses sustained by a constant mutational input (Durand et al. 2010, 2012, 2015) - values of mutational heritability ranged from 0.013 to 0.025 . We asked two main questions: How do mutations arise, spread, and reach fixation in populations where both drift and selection are extremely intense? How does the interplay between drift and selection influence the response to selection? To answer those questions, we confronted the observed phenotypic responses in Saclay DSEs to forward individual-based simulations that explicitly modeled the same selection and demographic scheme, and used theoretical predictions to measure deviations from expectations.

\section{Materials and Methods}

\section{Saclay Divergent selection experiments}

We have conducted two independent divergent selection experiments (Saclay DSEs) for flowering time from two commercial maize inbred lines, F252 and MBS847 (thereafter MBS). These experiments were held in the field at Universite Paris-Saclay (Gif-sur-Yvette, France). The selection procedure is detailed in Fig. S1 and Durand et al. (2010). Briefly, within each Saclay DSE, the ten earliest/ten latest flowering individuals were selfed at each generation to produce 100 offspring used for the next generation of selection within the Early/Late populations, so that 1000 plants were evaluated in each population. Following Durand et al. (2015), we designated as progenitor, a selected plant represented by its progenies produced by selfing and evaluated in the experimental design at the next generation. Seeds from progenitors from all generations were stored in cold chambers.

Within each population, we evaluated offspring of a given progenitor in four rows of 25 plants randomly distributed in a four-block design. Each block contained 10 rows representing the 10 progenitors. We applied a multi-stage pedigree selection. First the three earliest (latest) flowering plants within each row were selfed and their flowering time was recorded. This corresponded to 12 plants per progenitor, i.e. 120 plants per population. The second stage consisted in choosing 10 plants among the 120 on an index based on three criteria : flowering time, total kernel weight and pedigree. When two plants had the same flowering date, we chose the one with the highest kernel weight. In addition, we maintained two independent families within each population, i.e. two sub-pedigrees derived from two different progenitors in the ancestral $G_{0}$ population, and we never selected more than three plants from the same $G_{n-1}$ progenitor. Practically, each family was composed of three to seven progenitors at each generation. Altogether, we selected in each population 10 plants out of 1000 which corresponded to a selection intensity of $1 \%$.

We traced back the F252 and MBS pedigrees from generation $20\left(G_{20}\right)$ to the start of the divergent selection experiments, $G_{0}$. The initial MBS pedigrees encompassed four families: ME1 and ME2 for the MBS Early (ME) population, and ML1 and ML2 for the MBS Late (ML) population (Fig. S2). F252 Early (FE) population was composed of FE1 and FE2 families (Fig. S2). F252 Late populations genealogies were more complex: FVL families (F252 Very Late in Durand et al. (2015)) ended at generation 14 with the fixation of a strong effect allele at the $e I F-4 A$ gene (Durand et al. 2015). To maintain two families in F252 Late population, two families FL2.1 and FL2.2 were further derived from the initial FL2. These two families pedigrees are rooted in FL2 from a single $G_{3}$ progenitor (Fig. S2).

\section{Phenotypic data collection and empirical selection responses}

The same approach as Durand et al. (2015) was applied. Briefly, progenitor flowering dates, measured here as the number of days to flowering after sowing equivalent to $20^{\circ} \mathrm{C}$ days of development (Parent et al. 2010), were recorded as the 12 earliest or latest plants in their progeny at each generation of the Saclay DSEs. We used these records to investigate the response to selection treating each family independently. After correction of the phenotypic values $Z_{i j k l m n}$ for block effects, and year effects according to equation (1) of Durand et al. (2015) (so that $Y_{i j k l m}=Z_{i j k l m n}-\widehat{\text { Year }_{i}}-\widehat{\text { Block }_{i n}}$, in corresponding to the block effect $n$ in selection year $i$ ), the linear component $b_{j k}$ of the withinfamily response to selection was estimated using the following linear model:

$$
Y_{i j k l m}=\mu_{0}+b_{j k} \times \text { gener }_{i}+\varepsilon_{i j k l m}
$$

where $i$ stands for the year and corresponding generation of selection (so that gener ${ }_{i}$ takes values between 0 to 20 ) $j$ for the population (Late or Early), $k$ for the family within population (e.g ME1 or ME2), $l$ for progenitor within family, and $m$ for the plant measurements within progenitor (so that $\varepsilon_{i j k l m}$ corresponds to the residual variance due to differences between progenitor of the same generation, and family and plant effect). Finally, $\mu_{0}$ is the intercept corresponding to the average flowering time at generation $G_{0}$,

Family means and standard errors were also computed at each generation to represent families selection responses presented Fig. 1 (a). All the values were centered around 100 for comparison purposes with the simulated responses.

\section{Model framework}

We used forward individual-based simulations that explicitly modeled the same selection - proportion of selected individuals $=1 \%$ of the most extreme) - and demographic scheme variations in population size - as Saclay DSEs. This regime is referred to High-Drift High Selection intensity (HDHS). Initial $G_{0}$ simulation: We obtained our initial population by mimicking a classical selection scheme used to produce fixed maize inbred lines in industry. To do so, we started from an heterozygous individual that was selfed for eight generations in a single-seed descent design. An additional generation of selfing produced 60 offspring that were reproduced in panmixia for two generations to constitute the 60 individuals of the $G_{0}$ initial population. Therefore, we started our simulations with a small initial residual heterozygosity $(\leq 0.5 \%)$. $G_{1}$ simulation: Considering one 
which implied that:

$$
\mathbb{E}\left[a_{l}\right]=0 \text { and } \mathbb{V}\left[a_{l}\right]=\frac{2}{\lambda^{2}}
$$

Starting from a hybrid heterozygote at all $L$ loci, we computed of selfing and two generations of bulk. Selfing reduces the genic variance by $1 / 2$ each generation. In the absence of linkage disequilibrium, panmixia does not change allelic frequencies:

$$
\mathbb{E}\left(\sigma_{g+2}^{2}\right)=\frac{1}{2^{g}} \times L \times \mathbb{V}\left[a_{l}\right]=\frac{1}{2^{g}} \times L \times \frac{2}{\lambda^{2}}
$$

Therefore, to match the field estimate $\widehat{\sigma_{A_{0}}^{2}}=\mathbb{E}\left(\sigma_{g+2}^{2}\right)$, one could let

$$
\lambda=\sqrt{2 L \frac{1}{2^{g}} \frac{1}{\widehat{\sigma_{A_{0}}^{2}}} .}
$$

However, drift, linkage disequilibrium and mutation can lead to deviations from the expected value of the initial genetic variance. We therefore recalibrated all the allelic values at generation 0 to match the initial $\widehat{\sigma_{A_{0}}^{2}}$ additive variance. To do so, we multiplied all the allelic values by a corrective factor $k=$ $\sqrt{\widehat{\sigma_{A_{0}}^{2}} / \mathbb{V}\left(A_{0}\right)}$, where $\mathbb{V}\left(A_{0}\right)$ was the additive variance of our population $G_{0}$, calculated in multiallelic as $2 \times \sum_{l=1}^{L} \sum_{i=1}^{n} p_{i l} \alpha_{i l}^{2}$ with $n$ the number of alleles at locus $l, p_{i l}$ the frequency of the allele $\mathrm{i}$ at locus $1 . \alpha_{i l}$, its additive effect, is defined as in Lynch et al. (1998) (Chapter 4), so that after dropping subscript $l, \alpha_{i}=\mathbb{E}\left(G_{i j} \mid i\right)-\mu_{G}$, with $\mu_{G}$ the population genotypic mean, $\mathbb{E}\left(G_{i j} \mid i\right)$ the conditional expectation of the genotypic value of genotype $G_{i j}$ knowing $i$. So at $G_{0}, \mathbb{V}\left(A_{0}\right)=\widehat{\sigma_{A_{0}}^{2}}$.

Mutations occurred at each reproduction event. We drew the number of mutations per haplotype in a Poisson distribution of mean $L \times \mu$ where $\mu$ was the mutation rate per locus. Following Kimura (1979); Hill (1982a); Keightley (1994); Shaw et al. (2002); Piganeau and Eyre-Walker (2003), we drew the value of a mutation at a locus in a reflected exponential distribution of parameter $\lambda_{m u t}=2 \sqrt{(L \mu) / \sigma_{M}^{2}}$. We computed phenotypic values as the sum of all allelic values $a_{i l}(L \times 2)$ plus an environmental effect randomly drawn in a normal distribution of mean 0 and variance $\sigma_{E}^{2}$.

\section{Selection and drift regimes}

As control, we considered a model without selection (the No Selection regime, NS) where neutral evolution occurred in a population with the same census size and the same number of progenitors as in the selection model. At each generation, we randomly drew $1 \%$ of the individuals to form the next generation, instead of choosing them from their phenotypic values.

In addition, we considered an alternative drift regime, where we increased the census population size by a factor 10 , all other parameters remaining unchanged. This regime is referred to as the Low Drift regime (LD) where 50/5000 instead of 5/500 individuals were selected. Both Low Drift-High Selection (LDHS) and Low Drift-No selection (LDNS) were considered.

We performed 2000 independent simulations for each of the four families in each of the four regimes. All downstream analyses were carried out over all simulations, except when specified.

\section{Parameter calibration}

Our model encompassed three key parameters: the initial additive variance $\sigma_{A_{0}}^{2}$, the mutational variance, $\sigma_{M}^{2}$, and the environmental variance $\sigma_{E}^{2}$. We chose to sample parameter values in an inverse-gamma distribution with parameters (shape and scale) chosen such as (i) the expected means of the two inverse-gamma 


\section{Table 1 Initial variances parameters}

\begin{tabular}{lcccc}
\hline Parameter & Expectation & Variance & shape & rate \\
\hline$\sigma_{A_{0}}^{2}$ & 2.25 & 3.28 & 3.540 & 5.715 \\
$\sigma_{M}^{2}$ & $3.38 \times 10^{-2}$ & $7.27 \times 10^{-5}$ & 17.67 & 0.5626125 \\
$\sigma_{E}^{2}$ & 2.25 & 0.32 & 17.67 & 37.50075 \\
\hline
\end{tabular}

Genomic parameters were taken from the literature. In maize, Clark et al. (2005) estimated the nucleotidic substitution rate to $30 \times 10^{-9}$. We relied on the maize reference genome V4 (Jiao et al. 2017) to estimate an average mRNA length of 6000 (median=5197, mean=7314). Based on both estimates, we therefore considered a mutation rate per locus of: $\mu=6000 \times 30 \times 10^{-9}=$ $1.8 \times 10^{-4}$.

\section{Expected response, effective population size and time to the most recent common ancestor}

We computed the expected cumulative response after $t$ generations for haploid population as (Hill 1982b; Wei et al. 1996; Weber and Diggins 1990; Walsh and Lynch 2018):

$$
R(t) \approx N_{e} \frac{i}{\sigma_{P}}\left[t \sigma_{m}^{2}+\left(1-\mathrm{e}^{-\frac{t}{N_{e}}}\right)\left(\sigma_{A}^{2}(0)-N_{e} \sigma_{m}^{2}\right)\right]
$$

The effective population was the only parameter not explicitly defined in our simulations and is of crucial importance in the response to selection. We estimated $N_{e}$ following two approaches. First using the Time to the Most Recent Common Ancestor (TMRCA) from the standard coalescence theory for a haploid sample of size $k$ at generation $g$ (Walsh and Lynch 2018):

$$
\mathbb{E}\left(\mathrm{TMRCA}_{g}\right)=2 N_{e(g)}^{\text {Coal }} \times\left(1-\frac{1}{k}\right)
$$

Second, from the variance in offspring number (Crow and Kimura 1971; Durand et al. 2010), where $N_{e}$ can be computed as

$$
N_{e(g)}^{\operatorname{Var}(o)}=\frac{N-1}{\operatorname{Var}_{(\mathrm{g})}(\text { OffspringNumber })}
$$

In the simulations, $N_{e(g)}^{\text {Coal }}$ and $N_{e(g)}^{\operatorname{Var}(o)}$ were computed at generation $G_{20}$. We also computed the harmonic means between generations $G_{1}$ and $G_{20}$ and computed the whole distribution (in $2 N_{e}$ generations) of the Kingman coalescent TMRCA as (Tavaré 1984):

$f_{\mathrm{TMRCA}}(t)=\sum_{i=2}^{n} \frac{(2 i-1)(-1)^{i}(n(n-1) \ldots(n-i+1))}{n(n+1) \ldots(n+i-1)}\left(\begin{array}{l}i \\ 2\end{array}\right) \mathrm{e}^{-\left(\begin{array}{c}i \\ 2\end{array}\right) t}$
Fitness function and Kimura's expected fixed mutational DFE

Using diffusion equations, Kimura (Kimura 1962) predicts the fixation probability of a mutation of selective value $s(a)$ - with $a$ its allelic value - and initial frequency $p$ :

$$
P_{f i x}\left(s(a), p, N_{e}\right)=\frac{1-e^{-4 s(a) p N_{e}}}{1-e^{-4 s(a) N_{e}}} .
$$

When occurring, a new mutation arises during meiosis in one plant among the 500 of a family observed at a given generation. Hence, its effect on the phenotypic variance is negligible. Therefore, the plant carrying this newly arisen mutation was selected essentially independently of the new mutation. Consequently, the 5 individuals selected in one family comprised one heterozygote (Aa) bearing the mutation, and 4 homozygotes (aa). Each selected individual produced 100 progenies, so that the fitness effect of the mutation was evaluated at the next generation in a population of 500 plants where the frequency of the mutant allele was $p=1 / 10$ (Table 2 ).

Table 2 Fitness model

\begin{tabular}{lccc}
\hline Genotype & AA & Aa & aa \\
\hline Genotypic frequency & $1 / 20$ & $2 / 20$ & $17 / 20$ \\
\hline Fitness value & $w_{A A}$ & $w_{A a}$ & $w_{a a}$ \\
\hline Mutational effect & $a_{A A}=2 a$ & $a_{A a}=a$ & $a_{a a}=0$ \\
\hline
\end{tabular}

In this population, the distribution of flowering time resulted from a mixture of Gaussian distributions.

$$
f(x)=\sum_{k} \Pi_{k} f_{k}(x)
$$

where $f_{k}(x)$ is the flowering time distribution for plants with genotype $k \in A A, A a, a a$. As we selected $1 \%$ of the latest/earliest flowering plants, all selected plants did flower after the date $z$, computed as the $1 \%$ quantile of the mixture distribution. The selection effect $s(a)$ depended on the effect $a$ of the mutation on flowering time (Table 2). Indeed, the relative weight of homozygous mutants $A A$ among selected individuals was computed as:

$$
w_{A A}=\frac{1-F_{A A}(z)}{\sum_{k} 1-F_{k}(z)}
$$

Which leads to:

$$
s(a)=\frac{F_{a a}(z)-F_{A A}(z)}{1-F_{a a}(z)}
$$

The fixation probability $P_{f i x}\left(s(a), p, N_{e}\right)$ was computed as in (Eq. 12) using $s(a)$ (Eq. 15), $p=1 / 10$, and $N_{e(g)}^{\text {Coal }}$ for $N_{e}$. The mutational effect $a$ was drawn in a reflected exponential distribution of parameter $\lambda_{m u t}$ and density function $g_{\lambda_{m u t}}(a)$. Hence, the density of fixed mutations $h(a)$ was computed as:

$$
h(a)=\frac{g_{\lambda_{m u t}}(a) P_{f i x}\left(s(a), p, N_{e}\right)}{\int g_{\lambda_{m u t}}(x) P_{f i x}\left(s(x), p, N_{e}\right) d x} .
$$

Moreover, we recorded the simulated values $a_{\text {sim }}$ of each fixed mutation and computed the realized distribution $h_{o b s}(a)$ using kernel estimate methods. 


\section{Results}

In order to examine the evolution and fate of small selfing populations submitted to strong selection intensity, we investigated the dynamics of the response to selection under a High DriftHigh Selection intensity (HDHS) regime imposed on two divergent artificial selection experiments for flowering time in maize (Saclay DSEs). We compared experimental data to results of a simulation model specifically devised to mimic our experiments; and further computed when possible expectations from population and quantitative genetics theory.

Empirical response after $\mathbf{2 0}$ generations of selection In line with previous observations for the first 16 generations, we observed significant responses (Fig. 1 a, Tab. 3a, 3b) to selection after 20 generations in all families. Marked differences among families nevertheless characterized these responses. This is well exemplified in the Late F252 families where one family (FVL) responded very strongly with a mean shift of 11.32 Days to Flowering (DTF) after 13 generations, corresponding to a linear regression coefficient of $0.86 \mathrm{DTF} /$ generation (Tab. 3a). This family fixed a deleterious allele at $G_{13}$ and could not be maintained further (Durand et al. 2012). We examined two derived families from $G_{3}$ (Fig. S2), the FL2.1 and FL2.2. These families were shifted by 3.19 DTF and 2.60 DTF from the $G_{0}$ FL2 mean value for FL2.1 and FL2.2, respectively. These corresponded to a linear regression coefficient of 0.11 DTF/generation for FL2.1 and $0.12 \mathrm{DTF} /$ generation for FL2.2 (Tab. 3a). The selection response were more consistent for the two Early F252 families, with a shift after 20 generations of -4.27 DTF for FE1, and a shift of -5.34 DTF for FE2 (Tab. 3a). Considering MBS genetic background, the late/early MBS families were shifted by 8.64 DTF for ML1, and 11.05 DTF for ML2 (respectively -9.34 DTF for ME1 and -11.72 DTF for ME2), with linear regression coefficient of $0.24 \mathrm{DTF} /$ generation for ML1, and 0.46 DTF/generation for ML2 (respectively -0.41 DTF/generation for ME1 and $0.42 \mathrm{DTF} /$ generation for ME2) DTF (Tab. 3b).

Simulation model validation To parameterize our simulation model, we used priors: variance components were described by inverse-gamma distributions whose parameters were chosen following previously reported values for Saclay's DSEs Durand et al. (2010), mutation rate was taken from the maize literature (Clark et al. 2005), and the number of loci was set to $L=1000$ according to the large mutational target described for maize flowering time (Romero Navarro et al. 2017). In order to validate the parametrization of our model, we compared the observed MBS responses in all families to the simulated selection responses under HDHS regime. Because of the symmetry in the model construction and for simplicity, simulated results are described for late populations only. We recovered a simulated response with a mean genetic gain of $0.49 \mathrm{DTF} /$ generation (Fig. 1, Tab. 3c). Starting from a mean genotypic value of $100 \mathrm{DTF}$, the mean genotypic value was shifted by 13.0 DTF (SD: 5.2) after 20 generations. Our simulated response therefore closely matched the observed response ( $\mathrm{p}$-value not significant, Tab. 3b) indicating an accurate parametrization of our simulation model (Fig. 1), that captured the average selection response per generation of MBS. Note however, that inter-generational fluctuations were higher in the observations than in the simulations (Fig. 1).

We used simulations both to validate our model and to explore two drift intensities, High and Low. We used corresponding negative controls with No Selection (NS) which lead to four regimes: High Drift-High Selection intensity (HDHS, the default regime), High Drift-No Selection (HDNS), Low Drift-High Selection intensity (LDHS) and Low Drift-No Selection (LDNS).We formally tested the significance of our simulated response by comparing the linear response under HDHS to that obtained under HDNS. We were able to reject the null hypothesis of no selection response in $96.4 \%$ of the simulations under HDHS (P-value<0.05).

To investigate the impact of a ten-fold increase of the census population size on selection response, we contrasted HDHS to LDHS. Just like for HDHS, we obtained a significant response under LDHS with a mean genetic gain of 1.10 DTF/generation (Fig. 1, Tab. 3c). This gain was greater than the +0.035 DTF/generation (SD: 0.035) obtained for the LDNS control model, and we were able to reject the null hypothesis of no selection response in $100 \%$ of the simulations. The gain under LDHS corresponded to a shift of +24 DTF (SD: 6.2), which was substantially higher than that observed under HDHS. Hence multiplying the census population size of HDHS by 10 (LDHS) resulted in roughly doubling the selection response.

In sum, we validated the accuracy of our model by showing that the simulated response closely matched the observed response. We further demonstrated that selection triggered the response in all populations under both Low and High Drift. Finally, we confirmed our expectation that the selection response was higher in a Low Drift than in a High Drift regime.

Effective population size: We estimated coalescent effective population sizes $N_{e}$ from the standard coalescence theory (Eq: (9)) using a Wright-Fisher population of size 5 (HD) and 50 (LD) individuals. With 5 individuals, we expected a theoretical coalescence time around 8 generations, and with 50 individuals, around 98 generations (i.e. more than the number of simulated generations). Focusing on the last generation, our simulations provided estimates of the mean $G_{20}$ TMRCA of 7.6 generations under neutrality (NS) for HD, closely matching the theoretical expectation of 8 (Tab. 3c). Considering the LDNS simulations, theoretical expectations (98) largely exceeded the number of generations (20). In contrary, we found a mean $G_{20}$ TMRCA of 3.9 under HDHS, and 6.4 under LDHS. Fig. 2 shows the distribution of the TMRCA estimated at $G_{20}$ in the three regimes. Under HDNS, the distribution fits the expectation from Eq: (11). As compared to the neutral case, Fig. 2 also shows that both the high drift (HDHS) and low drift (LDHS) selection regimes display reduced TMRCA.

We next assessed the impact of selection on $N_{e}$ and compared different estimates, either based on TMRCA (Eq: (9)), or on the variance in offspring number (Eq: (10)), or on the cumulated response to selection (Eq: (8)). Values obtained are summarized in Tab. 3c. We found that in the absence of selection, $N_{e}$ estimated from the mean TMRCA were close to the actual number of reproducing individuals (4.8 for HDNS and $>10$ for LDNS), while they were much smaller under both selection regimes $(2.5$ for HDHS and 3.3 for LDHS). The observed differences between $N_{e(20)}^{\text {Coal }}$ and the harmonic mean of $N_{e(G 1-20)}^{\text {Coal }}$ revealed a strong influence of the first generation on the adaptive dynamics. When $N_{e}$ was computed from the variance in offspring number, estimates without selection (4.1 under HDNS and 42 under LDNS) were close to the actual number of reproducing individuals. Finally, $N_{e}$ estimates from the cumulated response to selection fell within the same range as the ones from the variance in offspring number in both selection regimes. In summary, most $N_{e}$ estimates were close to the actual number of reproducing individuals in the absence of selection but high selection intensity 
bioRxiv preprint doi: https://doi.org/10.1101/2020.12.22.423930; this version posted July 14, 2021. The copyright holder for this preprint (which was not certified by peer review) is the author/funder, who has granted bioRxiv a license to display the preprint in perpetuity. It is made available under aCC-BY-NC-ND 4.0 International license.

(a) Observed High Drift-High Selection

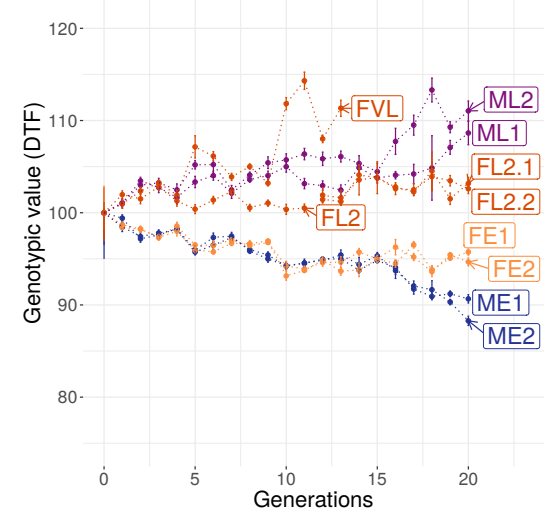

(b) High Drift-High Selection

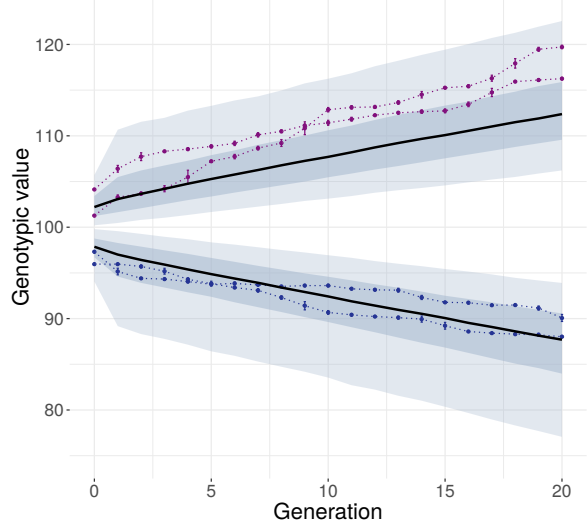

(d) High Drift-No Selection

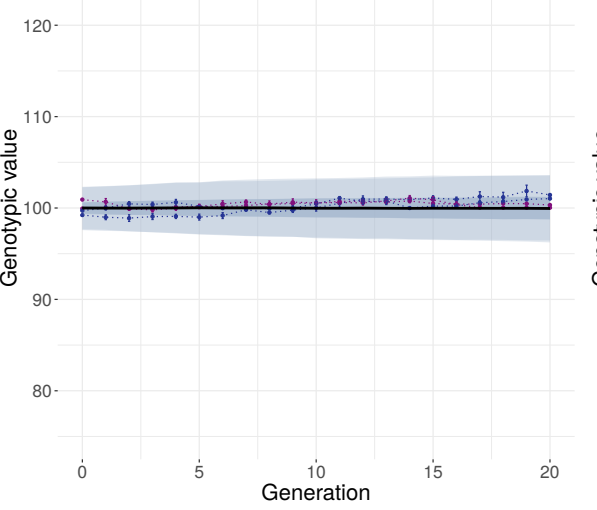

Simulated

(c) Low Drift-High Selection

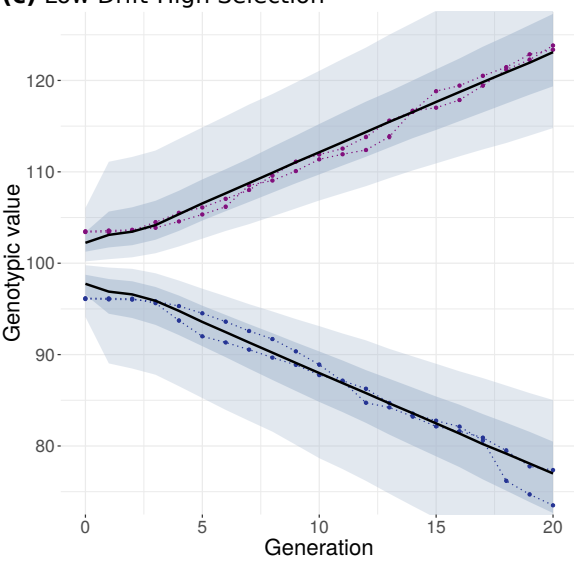

(e) Low Drift-No Selection

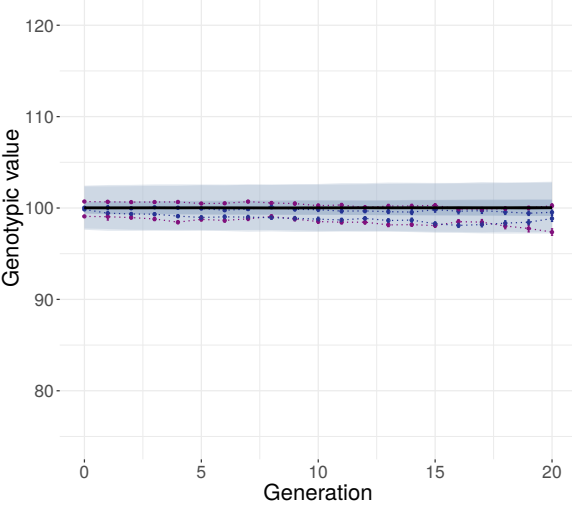

Figure 1 Observed and simulated selection response. Selection response is visualized by the evolution of the mean genotypic values of the selected progenitors per family (expressed in Days To Flowering, DTF) across generations in observed (a) and simulated (b-e) data. Observed genotypic values correspond to mean phenotypic values corrected for environmental effects . In (a), red/orange corresponds to late/early flowering F252 families, while violet/blue corresponds to late/early flowering MBS families. All families were centered around 100, and Vertical bars correspond to \pm 1 genotypic standard error around the mean. We simulated four regimes with the parameters calibrated from the MBS observed response: High Drift-High Selection intensity (b), Low Drift-High Selection (c), High Drift-No Selection (d), Low Drift-No Selection (e). Violet/blue color identifies late/early population. In each population, the black line represents the evolution of the median value over 2000 simulations of the family genotypic mean. The shaded area corresponds to the $5^{\text {th }}-95^{\text {th }}$ percentiles (light blue) and to the $25^{t h}-75^{t h}$ percentiles (dark blue). In addition, two randomly chosen simulations are shown with dotted lines

strongly reduced $N_{e}$ estimates.

Stochasticity in the response to selection: We addressed the qualitative nature of selection response focusing on its linearity. To do so, we measured in each family the average genetic gain per generation over 2000 simulations by fitting a linear regression model. The average genetic gain was $0.49 \mathrm{DTF} /$ generation under HDHS, and 1.1 DTF/generation under LDHS (Tab. 3c). Associated $R^{2}>0.95$ indicated an accurate fit of the data to the linear model. Yet, large standard deviations around these estimates (0.2 and 0.27 for HDHS and LDHS, respectively) pointed either to high stochasticity or a non-linear response. Single simulations indicated non-linear response (Fig. S3). Noteworthy, a strong response was observed between $G_{0}$ and $G_{1}\left(G_{0} G_{1}\right.$ Fig. S3) with similar values in HDHS and LDHS, around 1.6 DTF/generation (Tab. 3c). Subsequently, simulations displayed discontinuities with abrupt changes of slopes at some generations, a signal com- patible with the fixation of new mutations (Fig. S3). In order to characterize such discontinuities, we fitted a linear segmentation regression on individual simulations from $G_{1}$ and onwards. We estimated the number of breakpoints (i.e. slope changes), the corresponding slopes, and the first and greatest slope based on AIC minimization (Durand et al. 2010). The first slope described an average gain of $0.59 \mathrm{DTF} /$ generation in the HDHS regime, and almost twice (0.96 DTF/generation) in the LDHS regime (Tab. 3c). These values were lower than those observed in $G_{0} G_{1}$.

Those results are consistent with a $G_{0} G_{1}$ response resulting from the recruitment of initial genetic variance, independently of the population size, and a later response based on mutational variance being less effective in small than in large populations. To confirm those results, we performed a principal component analysis (PCA) and explored correlations between input parameters: initial additive genetic variance $\sigma_{A_{0}}^{2}$, mutational variance 

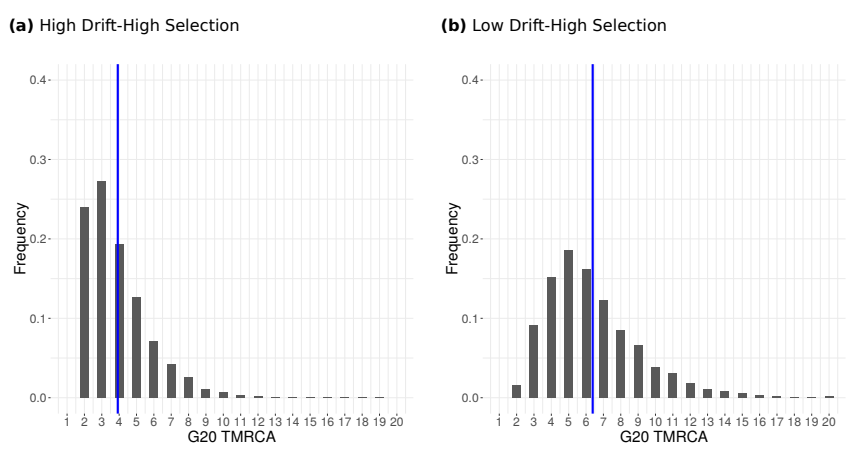

(c) High Drift-No Selection

(d) Low Drift-No Selection

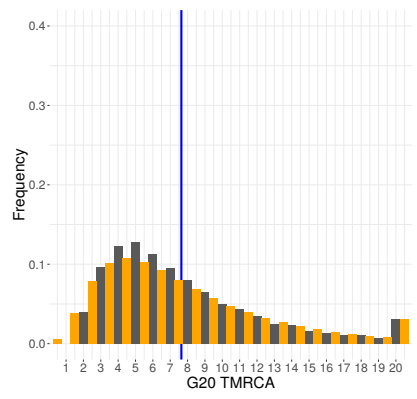

Figure 2 Frequency distribution of the Time to the Most Common Ancestor of progenitors constituting the last simulated generation. $G_{20}$ TMRCA distribution (in grey) was obtained under HDHS (a), LDHS (b), HDNS (c) with mean TMRCA indicated as a blue vertical line. In (c), we plotted in gold the theoretical expectation of TMRCA distribution following Eq: (11). Note that under LDNS, theoretical expectations for TMRCA reached 98 generations, while our simulations were run for 20 generations. We therefore discarded the corresponding graph.

$\sigma_{M}^{2}$ and residual variance $\sigma_{E}^{2}$, and descriptors of the response to selection : $G_{0} G_{1}$ response, number of breakpoints, first slope and greatest slope. In line with our interpretation, irrespective of the selection regime, $\sigma_{A_{0}}^{2}$ positively correlated with $G_{0} G_{1}$, and $\sigma_{M}^{2}$ positively correlated with the first (after $G_{1}$ ) and greatest slope (Fig. S4). Note that this stochastic process of mutation occurrence and fixation resulted in large differences among replicates, as illustrated by the breadth of the response (shaded areas in Fig. 1).

Evolution of genetic diversity: Because of the well-established role of standing variation in selection response, we focused on its temporal dynamics. Standing variation in our experiment consisted in residual heterozygosity found in the initial inbred lines. Starting with a mean residual heterozygosity of $3.0 \times 10^{-3}$ at $G_{0}$ (Tab. 3c), we observed a consistent decrease throughout selfing generations until the mutation-drift-equilibrium was reached (Fig. S5). The mean values reached $\approx 7.0 \times 10^{-4}$ at $G_{20}$ without selection, and $\approx 8.0 \times 10^{-4}$ with selection (Tab. 3 c) irrespective of the census population size.

Concerning the number of polymorphic loci, a mutationdrift-equilibrium was reached in all cases except for the LDNS selection regime (Fig. S6). The equilibrium value depended on the census population size: around 6 polymorphic loci with high drift (HDHS and HDNS), 40 polymorphic loci under LDHS, and $>66$ polymorphic loci after 20 generations under LDNS (Tab. 3c (c) and Fig. S6). Altogether, our results show that the mean heterozygosity was affected neither by drift, nor by selection, but instead by the mutation rate. On the contrary, the number of polymorphic loci depended on the census population size.
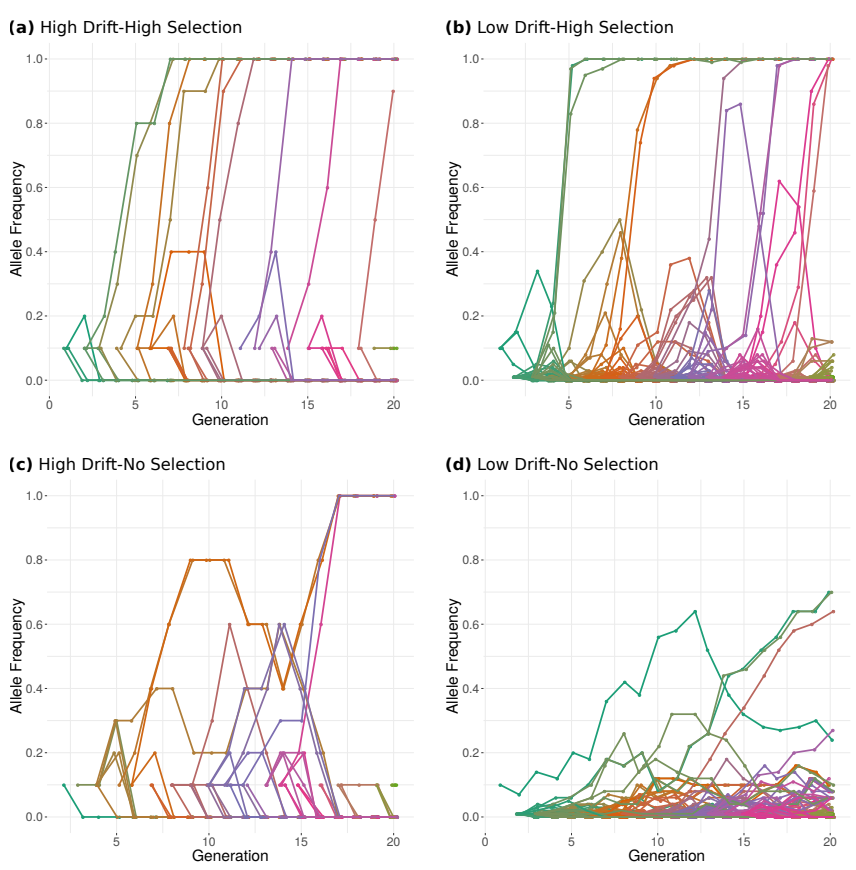

Figure 3 Evolution of allele frequencies within families under four simulated regimes. Examples of mutational fates are given for HDHS (a), LDHS (b), HDNS (c), LDNS (d). Mutations are recorded only when occurring in one of the selected progenitors, and corresponding frequencies are computed over all selected individuals. For example under High Drift regimes, the initial frequency of a mutation occurring in any given progenitor within a family is $1 \div(2 \times 5)$ as 5 diploid individuals are selected at each generation. Under Lower Drift regimes, the mutation initial frequency equals $1 \div(2 \times 50)$.

The dynamics of de novo mutations: Evolution of frequencies of new mutations revealed three fates: fixation, loss, and rare replacement by de novo mutation at the same locus. The four regimes strikingly differed in their mutational dynamics (Fig. 3). Under HDHS, most mutations quickly reached fixation (3.8 generations), with an average of 7.7 fixed mutations/population in 20 generations (Tab. 3c). The corresponding Low Drift regime (LDHS) displayed longer fixation time 5.9 generations, and an average of 10 fixed mutations/family (Tab. 3c). Regimes without selection tended to exhibit a depleted number of fixed mutations, with no fixation under LDNS after 20 generations. Variation around the mean fixation time was substantial across all regimes (Fig. S7). In sum, HDHS was characterized by the fast fixation of new mutations whose direction corresponded to the direction of selection: $53 \%$ were fixed within 2 to 3 generations which contrasted to $15 \%$ under LDHS or $17 \%$ under HDNS. Selection therefore increased the number of fixed mutations while decreasing their fixation time.

Effects of mutations: Beyond fixation time, a key aspect of our work was to investigate the impact of drift and selection on the type of fixed mutations, best summarized by their genotypic 
(a) High Drift-High Selection

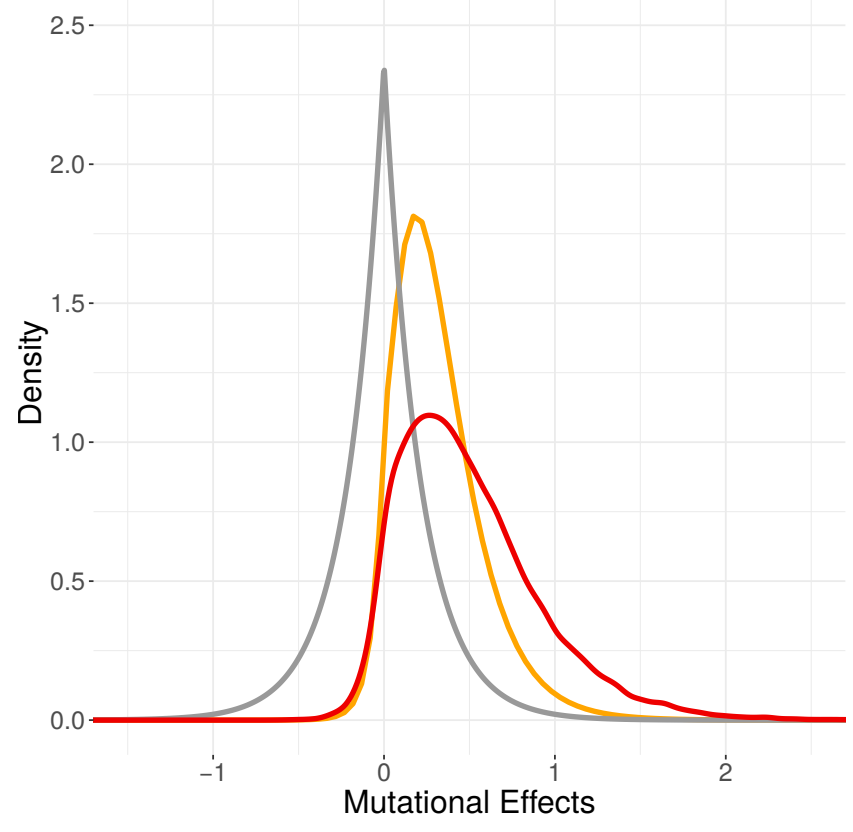

(b) Low Drift-High Selection

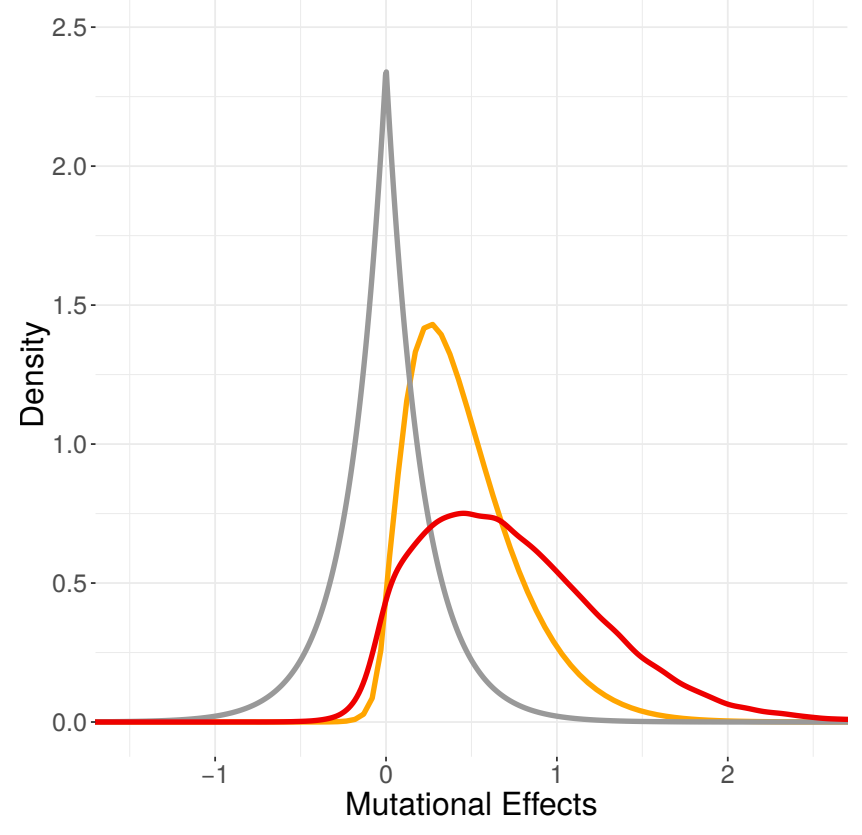

Figure 4 Distribution of effects of de novo and fixed mutations under High Selection intensity regimes. Density distributions for the HDHS (a) and the LDHS (b) regime are shown for all de novo mutational effects in grey - reflected exponential distribution - , and fixed mutations over 2000 simulations in red. Theoretical expectations from (Eq: 16) are plotted in gold. effects. In order to do so, we compared the distribution of incoming de novo mutations to that of fixed mutations. We observed a strong depletion of deleterious mutations together with a striking enrichment in beneficial mutations under the two Selection regimes, HDHS (quantile 5\%=-0.02, median value $=0.43,95 \%$ quantile $=1.3$ ) and LDHS (quantile $5 \%=0.011$, median value $=0.66$, 95\% quantile=1.7) (Fig: 4). We also derived a theoretical expectation from Kimura's allele fixation probability using the selection coefficient computed in the case of truncation selection (Eq: 16). Accounting for the specificities of our selection procedure we found under both selection regimes, a slight excess of detrimental mutations, and a large excess of beneficial mutations as compared to Kimura's predictions. Note however that, comparatively, the excess of detrimental mutations in the simulations compared to theoretical expectations was more reduced under HDHS than under LDHS (Fig: 4).

As expected, selection generated a relation between the average size of a mutation and its time to fixation : the higher the effect of the mutation, the lower the time to fixation (Fig. 5 (a) and Fig. 5 (b)). Comparison between HDHS and LDHS revealed interesting features: under high drift, the average effect of mutations fixed was lower and variance around mutational effects tended to decrease correlatively with fixation time so that large size mutations were all fixed during the first generation while they persisted at subsequent generations under Low Drift (Fig. 5 (a) and Fig. 5 (b)).

In sum, our two selection regimes lead to an enrichment of beneficial mutations. Compared with LDHS, HDHS regime fixed fewer detrimental mutations but the average effect of fixed beneficial mutations was smaller.
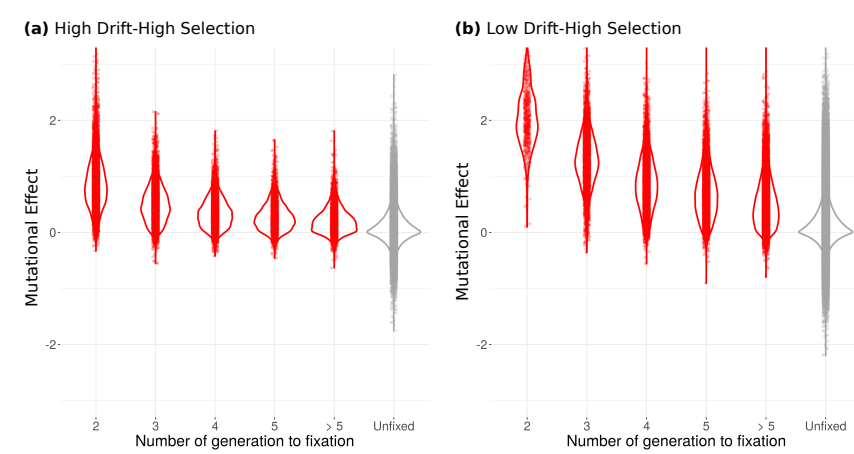

(c) High Drift-No Selection

(d) Low Drift-No Selection

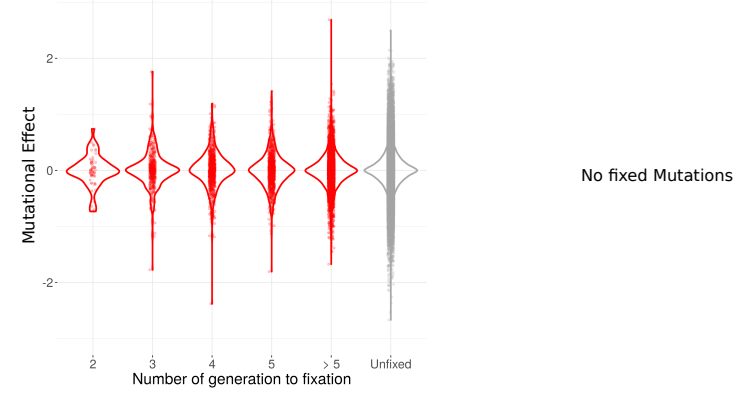

Figure 5 Violin plots of raw mutational effects according to fixation time under three simulated regimes. Plots are indicated for fixed (red) and lost (grey) mutations under HDHS (a), LDHS (b) and HDNS (c). Note that under LDNS, we obtained very few fixed mutations so that we were unable to draw the corresponding distribution.

Covariation between mutational and environmental effects: A puzzling observation was that normalizing raw mutational effects by the environmental standard deviation of selected individuals translated into a distortion of the distribution so that the median 9 10 11 12 13 14 7 18 19 20 21 22 24 25 26 28 29
30

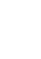




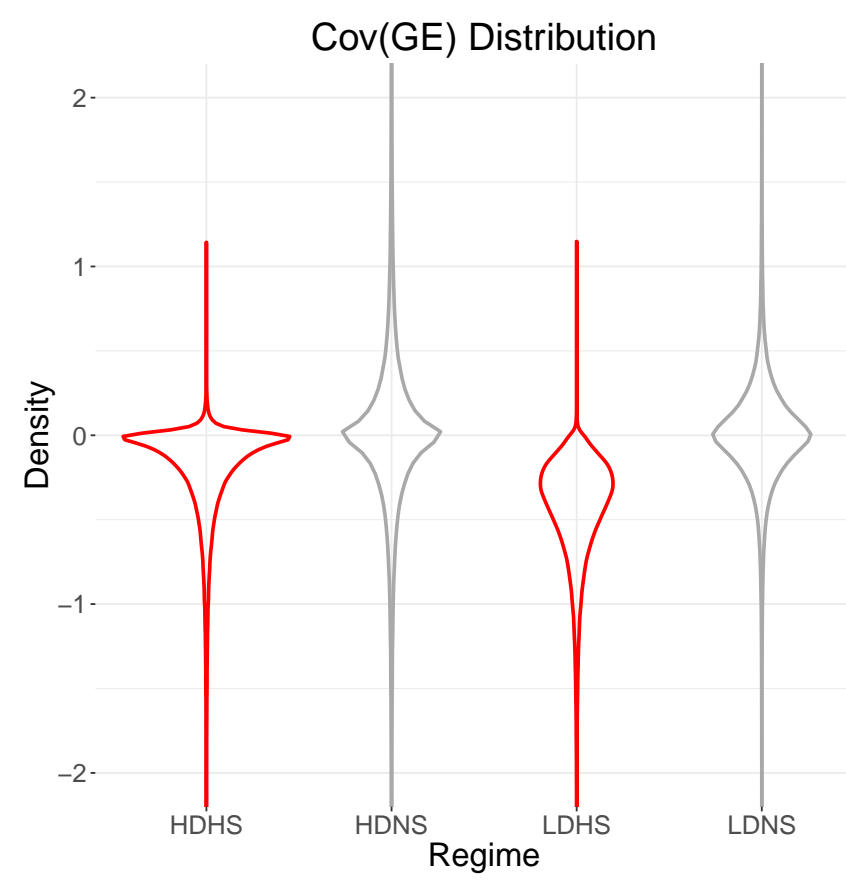

Figure 6 Violin plots of $\operatorname{Cov}\left(G_{\mid \text {selected }}, E_{\mid \text {selected }}\right)$ under four simulated regimes. Violin plots were computed over 2000 simulations and 4 families, four families and across all generations under regimes with High Selection intensity in red (HDHS, LDHS), and regimes with No Selection in grey (HDNS, LDNS).

value of fixed effects increased by 0.29 (from 0.43 to 0.72 ) under HDHS and by 0.2 under LDHS (Tab. 3 and Fig. S8). Similarly, 95\% quantile increased by 1.2 (from 1.3 to 2.5 ) under HDHS and 0.66 (from 1.7 to 2.4 ) under LDHS. Hence, normalization distortion resulted in much more similar fixed mutations effects distribution under HDHS and HDNS. This was due to a non-zero negative genetic-environment covariance in selected individuals. Indeed, conditioning on the subset of selected individual, we obtained negative estimate of $\operatorname{Cov}\left(G_{\mid \text {selected }}, E_{\text {|selected }}\right)$ both under HDHS and LDHS, with a median value (respectively $5 \%$ and $95 \%$ quantile) of -0.11 (respectively -0.88 and 0.029 ) under HDHS and -0.37 (respectively -1.1 and -0.072) under LDHS. In contrast, with no selection, values of randomly chosen individual $\operatorname{Cov}\left(G_{\mid \text {random }}, E_{\mid \text {random }}\right)$ were centered around 0 as expected. The evolution of $\operatorname{Cov}\left(G_{\mid \text {selected }}, E_{\mid \text {selected }}\right)$ through time (Fig. S9) evidenced a high stochasticity among generations but no temporal autocorrelation (Fig. S9). In other words, because of the negative correlation between residual environmental effects and genetic effects induced by selection, mutational effects tightly depended on their environment of selection.
Our main motivation was to explore how such a combination of unusual conditions, at the limits of parameters boundaries of classic models, can sustain the long-term maintenance of additive genetic variation and a significant selection response with no observed load (annual field observations). In this purpose we (1) devised forward individual-based simulations that explicitly modeled our Saclay DSEs and were calibrated on observed values of initial, mutational and environmental variances, and (2) relied on theoretical predictions to investigate the interplay of evolutionary forces and patterns associated with fixation of mutations.

The broadness of the mutational target sustains long-term mutational input The three determinants of the observed selection response were best summarized by three variance components namely, the initial additive variance $\sigma_{A 0}^{2}$, the mutational variance $\sigma_{M}^{2}$, and the environmental variance $\sigma_{E}^{2}$ (Fig: S4). Quantitatively, we demonstrated the importance of both initial standing variation and the necessity of a constant mutational input to explain the significant selection response in the two Saclay DSEs (Fig: 1 \& S4). This result was consistent with previous reports (Durand et al. 2010, 2015) and showed that the first selection response between $G_{0}$ and $G_{1}$ was correlated with $\sigma_{A 0}^{2}$, while response in subsequent generations was mainly determined by $\sigma_{M}^{2}$ (Fig: S4). In our simulations, we chose initial values for variance components that closely matched previous estimates in the Saclay DSE derived from the MBS inbred line (Durand et al. 2010). The small value for initial additive variance came from the use of commercial inbred lines in our experimental evolution setting. It sharply contrasted with more traditional settings where distant genetic material and crosses are often performed to form an initial panmictic population on which selection is applied (Kawecki et al. 2012). While crucial in the first generation (Fig: S4), $\sigma_{A 0}^{2}$ was quickly exhausted. Hence, we showed that the long-term selection response was sustained by a strong mutational variance. The chosen variance $\mathbb{E}\left(\sigma_{M}^{2}\right)=3.38 \times 10^{-2}$, corresponded to an expected mutational heritability of $\mathbb{E}\left(\sigma_{M}^{2} / \sigma_{E}^{2}\right) \approx \mathbb{E}\left(\sigma_{M}^{2}\right) / \mathbb{E}\left(\sigma_{E}^{2}\right)=1.5 \times 10^{-2}$ (in units of residual variance per generation). This value, observed in our setting for flowering time, stands as a higher bound to what was previously described in other species/complex traits (Keightley 2010; Walsh and Lynch 2018).

$\sigma_{M}^{2}$ is intimately linked to the broadness of the mutational target, a key parameter of our setting. While decreasing the genomic mutation rate (U) - either by modifying the number of loci or by the per-base mutation rate - we observed at first sight a stronger average response to selection (Fig. S10). This is because incoming de novo mutational effects increase correlatively with decreasing $\mathrm{U}$, to maintain $\sigma_{M}^{2}$ constant. Note that stochasticity of the response is boosted by scarcity of strong effect mutations that are preferentially fixed (Fig. S10). If instead of conditioning on $\sigma_{M}^{2}$, we conditioned on the distribution of incoming de novo mutational effects, responses under low values of $U$ were drastically reduced (Fig. S10). These results further highlight that adaptive evolution results from a subtle balance between mutation, drift and selection.

We implemented an additive incremental mutation model (Clayton and Robertson 1955; Kimura 1965; Walsh and Lynch 2018). This model assumed non-limiting mutational inputs, and has been shown to be particularly relevant in systems where, just like ours, effective recombination is limited (Charlesworth 1993; Walsh and Lynch 2018). Alternative model such as the House Of Cards (HoC) that sets random allelic effect upon oc- 
bioRxiv preprint doi: https://doi.org/10.1101/2020.12.22.423930; this version posted July $14,2021$. The copyright holder for this preprint (which was not certified by peer review) is the author/funder, who has granted bioRxiv a license to display the preprint in perpetuity. It is made available under aCC-BY-NC-ND 4.0 International license.

Table 3 Descriptive statistics of the selection response dynamics in observed F252 genetic background (a), observed MBS genetic background (b) and the 4 simulated regimes (c).

(a) HDHS observed in F252 genetic background

\begin{tabular}{|llllll|}
\hline F252 families: & FE1 & FE2 & FVL (G13) & FL2.1 & FL2.2 \\
\hline Cumul. Resp. in DTF & -4.27 & -5.34 & 11.32 & 3.19 & 2.60 \\
Linear Regression Coefficient (SD) & $-0.21(0.048)$ & $-0.22(0.037)$ & $0.86(0.17)$ & $0.11(0.04)$ & $0.12(0.035)$ \\
Adjusted R-squared & 0.49 & 0.63 & 0.65 & 0.23 & 0.34 \\
Linear regression p-value & 0.000269 & 1.074 e-05 & 0.000305 & 0.016 & 0.00353 \\
\hline
\end{tabular}

(b) HDHS observed in MBS genetic background

\begin{tabular}{|c|c|c|c|c|}
\hline MBS families: & ME1 & ME2 & ML1 & ML2 \\
\hline Linear Regression Coefficient (SD) & $-0.41(0.03)$ & $-0.42(0.04)$ & $0.24(0.05)$ & $0.46(0.06)$ \\
\hline Adjusted R-squared & 0.89 & 0.84 & 0.57 & 0.76 \\
\hline Linear regression $p$-value & $1.01 \mathrm{e}-10$ & $3.52 \mathrm{e}-09$ & $4.46 \mathrm{e}-05$ & $1.56 \mathrm{e}-07$ \\
\hline
\end{tabular}

(c) Simulated regimes ${ }^{b}$

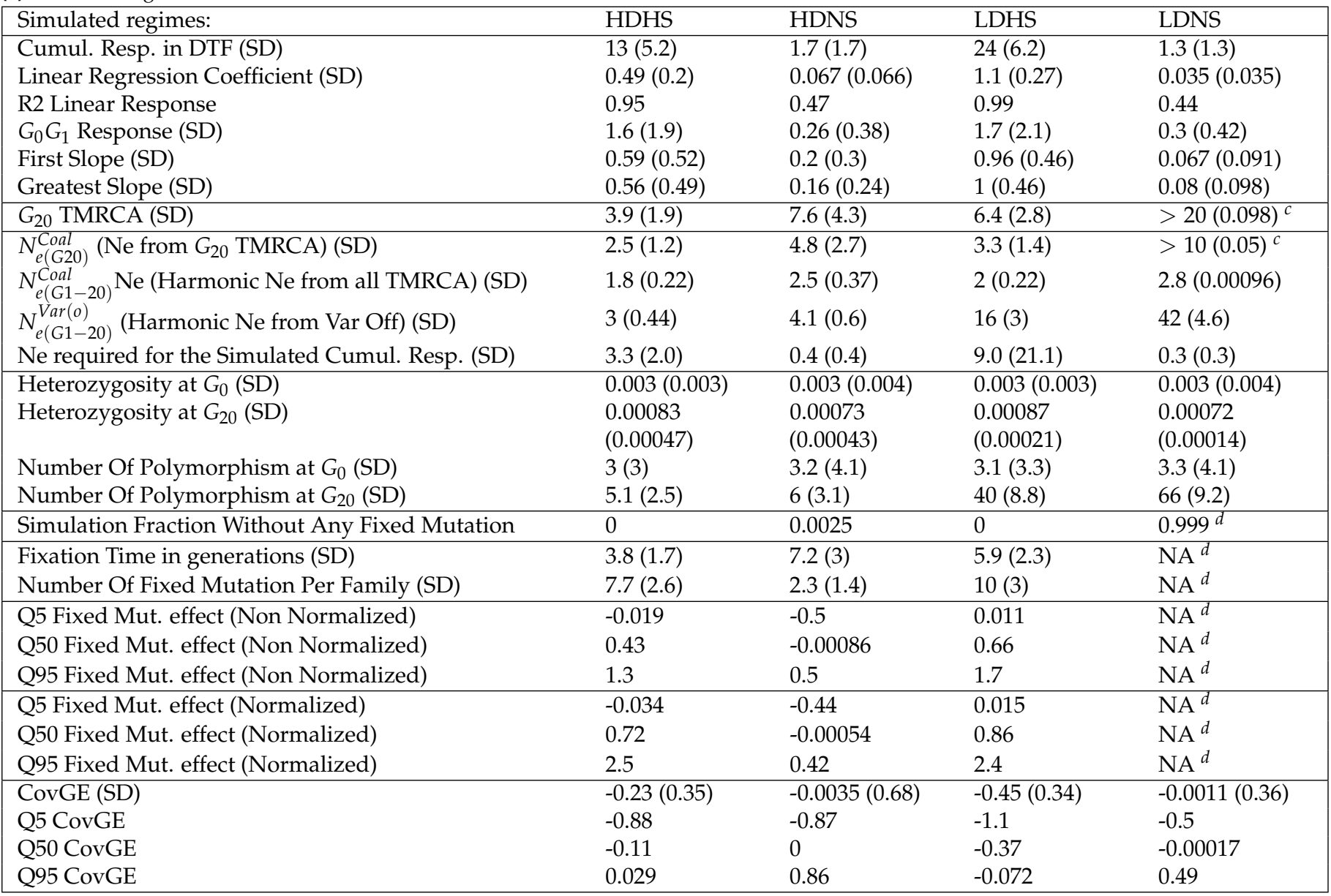

${ }^{a}$ P-values are computed from the distribution of cumulative response under simulated HDHS.

${ }^{b}$ All values are computed as the mean (SD or quantile respectively, when specified) over 2000 simulations.

${ }^{c}$ Under LDNS, we expected a neutral coalescent time around 98 generations well beyond the 20 simulated generations, which provided highly biased $G_{20}$ TMRCA and $N_{e}$ estimators.

${ }^{d}$ Under LDNS, we obtained very few fixed mutation so that we were unable to compute the corresponding statistics. 
currence of a new allele (Kingman 1978; Turelli 1984) — rather than adding effects incrementally - would have likely resulted in smaller estimate of $\sigma_{M}^{2}$ (Hodgins-Davis et al. 2015). Whether the incremental model or the $\mathrm{HoC}$ or a combination of both such as the regression mutation model (Zeng and Cockerham 1993) was better suited to mimic our Saclay DSEs is an open question. However several lines of evidence argue in favour of a nonlimiting mutational input in our setting. First, the architecture of maize flowering time is dominated by a myriad of QTLs of small additive effects (Buckler et al. 2009). Over 100 QTLs have been detected across maize lines (Buckler et al. 2009), and over 1000 genes have been shown to be involved in its control in a diverse set of landraces (Romero Navarro et al. 2017). Second, in Saclay DSEs alone, transcriptomic analysis of apical meristem tissues has detected 2,451 genes putatively involved in the response to selection between early and late genotypes, some of which being interconnected within the complex gene network that determines the timing of floral transition (Tenaillon et al. 2018). This suggests that not only the number of loci is considerable, but also that their connection within a network further enhances the number of genetic combinations, and in turn, the associated phenotypic landscape.

The breadth of the mutational target is a key parameter for adaptation (Höllinger et al. 2019). Altogether, our results suggest that despite a small number of segregating loci (Tab. $3 \mathrm{c}$ and Fig. S6) expected under HDHS, adaptive variation was continuously fueled by a large mutational target which translated into a long-term selection response. In other words, the large mutational target compensates for the small population sizes, and triggers the long-term maintenance of adaptive diversity at the population level after the selection-drift-mutation equilibrium is reached, i.e. after three to five generations. Noteworthy the expected level of heterozygosity in our controls (No Selection models, NS) corresponded to neutral predictions (Crow and Maruyama 1971; Kimura 1969). our estimates of $N_{e}$ - based on coalescence times computed from the known genealogical structure - allow to account for these effects. However, this is not without drawback: TMRCA estimates were much shorter than expected, a result consistent with the occurrence of multiple merging along pedigrees, i.e. multiple individuals coalescing into a single progenitor. In fact, multiple merger coalescence models may be better suited to describe rapid adaptation than the Kingman coalescent (Neher 2013).

Both fixation time and probability depend on the selection coefficient $s$ and the initial frequency of the mutation in the population. In our setting, conditioning on its appearance in the subset of selected individuals, the initial frequency of a mutation was 0.10 , which was unusually high and translated into selection and drift exerting greater control over mutations. In contrast, in more traditional drift regimes, even when an allele is strongly selected $\left(2 N_{e} s \gg 1\right)$, drift dominates at mutation occurrence, i.e. with two absorbing states for allele frequency near zero and one (Walsh and Lynch 2018). Under HDHS regime, selection induces repeated population bottlenecks that change the structure of the pedigrees and translate into a decrease in effective population size compared to the HDNS regime. Drift and selection can therefore not be decoupled, and they do not act additively.

High stochasticity promotes the fixation of small effect mutations Interplay between drift and selection promoted stochasticity in our setting, which manifested itself in various ways : (i) through the selection response, with different families exhibiting contrasting behaviors, some responding very strongly and others not (Fig. 1); (ii) through the dynamics of allele fixation (Fig. 2 \& 3); and (iii) through the distribution of $\operatorname{Cov}(G E)$ (Fig. 6). Stochasticity tightly depends on census population size (Hill 1982a,b). Unexpectedly, however, we found a benefice to stochasticity as illustrated by a bias towards the fixation of advantageous mutations compared with the expectation (Fig. 4). Comparison of the distributions of the mutational raw effects indicated that, among advantageous mutations, a greater proportion of those with small effects were fixed under the High Drift than under the Low Drift regime (Fig. 4 (a) versus (b) ). This result echoes those of Silander et al. (2007), who showed - using experimental evolution with bacteriophage - that fitness declines down to a plateau in populations where drift overpowers selection. The authors note: "If all mutations were of small effect, they should be immune to selection in small populations. This was not observed; both deleterious and beneficial mutations were subject to selective forces, even in the smallest of the populations.".

What are the underlying mechanisms behind this fixation bias? We found a negative covariance between selected genotypes and their corresponding micro-environmental values, that modified the mutational effect to an apparent mutational effect perceived in the particular micro-environment. The negative $\operatorname{Cov}(G E)$ arose mechanically from selection of two independent random variables, whatever the sampling size as illustrated in Fig. S11 and Fig. S12. This effect evokes the so-called Bulmer effect (Bulmer 1971), that causes a reduction of genetic variance due to the effect of selection on the covariance between unlinked loci. Interestingly, under the High Drift regime, we observed a less negative $\operatorname{Cov}(G E)$ on average than with a 10 times higher census size (Low Drift). This translated, after dividing by the environmental standard deviation of selected individuals, to a greater apparent effect of small mutations under the High Drift regime. In other words, High Drift-High Selection intensity tends to magnify mutational effects from an environmental per- 
spective. In support of this explanation, normalization by the environmental standard deviation actually erased the difference between the two distributions of mutational effect (under low and High Drift, Fig. S8). Unlike the Bulmer effect however, this one was restricted to the generation of mutation occurrence, but favored long-term fixation of slightly advantageous mutations by a transient increase of their frequency. Because of a significant variance of $\operatorname{Cov}(G E)$, this effect on small effect mutation fixation was mostly stochastic. Therefore, we interpreted the fixation of a high proportion of slightly beneficial mutations, and their significant contribution to selection response, by the less efficient exploration of the initial distribution per simulation (increasing their prevalence) but the stochastic "help" of a lesser negative $\operatorname{Cov}(G E)$.

Just like $\operatorname{Cov}(G E)$, epistatic interactions may further exacerbate stochasticity Dillmann and Foulley (1998). While we showed that de novo mutations are fixed sequentially and therefore rarely interact, additive effects of new mutations tightly depend on the genetic background in which they occur (Plucain et al. 2014), and mutational history modulates the amount of additive genetic variance (Hill et al. 2008). While we have not accounted for them, epistatic interactions may hence result in a distortion of the distribution of mutation effects. Epistasis can also generate asymmetrical selection responses (Lynch et al. 1998; Keightley 1996). Because the two initial inbred lines, F252 and MBS, have been intensively selected for earliness (CamusKulandaivelu et al. 2006; Rebourg et al. 2003), we expect in our setting a diminishing return of mutational effects in the Early populations and, conversely, mutations of high effect in the Late populations (Durand et al. 2010). Any new mutant occurring within these 'early' genetic backgrounds, can either constrain or accentuate phenotypic effects, depending on direction of selection. Altogether, epistasis could explain some discrepancies between observations and simulations, such as the high stochasticity in Saclay DSEs.

\section{Deficit of fixation of deleterious mutations suggests a limited cost of} selection As expected, we observed that selection decreased the number of segregating polymorphic loci at equilibrium compared to regimes without selection (Tab. 3). Interestingly however, this effect was reduced for small population size. Under High Drift, selection induced an average loss of a single polymorphism at equilibrium (HDHS vs. HDNS, Tab. 3) while under the Low Drift regime over 20 polymorphisms were lost (LDHS vs. LDNS, Tab. 3). A similar trend was recovered at the mutation fixation level where on average 7.7 mutations were fixed under the High Drift-High Selection intensity and only 10 under Low Drift-High Selection intensity. In other words, the 10-fold population increase did not translate into a corresponding increase in the number of segregating and fixed mutations, as if there was a diminishing cost with decreasing population size. Under High Drift (/Low Drift), at each generation 500 (/5000) offspring of $2 \times 1000$ loci were produced. Considering a mutation rate per locus of $6000 \times 30 \times 10^{-9}$, (i.e. (Clark et al. 2005)), it translated into 180 mutations events (/1800 mutations events). However most mutations are lost as only mutations occurring in the subset of selected individuals survive. The initial frequency of a mutation in this subset, i.e of size 5 or 50 , is $1 / 10$ under High Drift and $1 / 100$ under Low Drift. In the former, the interplay between the initial frequency and selection intensity allows a better retention of beneficial mutations of small effect (Fig. 4) than in the latter. Interestingly at equilibrium, we also observed a higher level of residual heterozygosity with selection than without, irrespective of population size, suggesting a small impact of selection in the long-term heterozygosity maintenance. Overall, our High Drift-High Selection intensity regime maintains a small, but sufficient number of polymorphisms for the selection response to be significant.

Our selection response evidenced a deficit of fixation of deleterious mutations and hence a modest genetic load (Fig. 4 and S8). We identified three reasons behind this observation. Firstly, in our design, the selection intensity of $1 \%$ was applied on the trait. Hence, in contrast to the infinitesimal model for which a high number of polymorphic loci are expected to individually experience a small selection intensity, selection intensity was "concentrated" here on a restricted number of loci, i.e. those for which polymorphisms were segregating. Secondly, we applied truncation selection whose efficiency has been demonstrated (Crow and Kimura 1979). The authors noted: "It is shown, for mutations affecting viability in Drosophila, that truncation selection or reasonable departures therefrom can reduce the mutation load greatly. This may be one way to reconcile the very high mutation rate of such genes with a small mutation load." Thirdly, the lack of interference between selected loci in our selection regime may further diminish the selection cost (Hill and Robertson 1966). Reduced interference in our system is indeed expected from reduced initial diversity and quick fixation of de novo mutations. Whether natural selection proceeds through truncation selection or Gaussian selection is still a matter of debate (Crow and Kimura 1979). Measuring the impact of these two types of selection on the genealogical structure of small populations including on the prevalence of multiple merging branches will be of great interest to better predict their fate.

This under-representation of deleterious variants echoes with empirical evidence that in crops, elite lines are impoverished in deleterious variants compared to landraces owing to a recent strong selection for yield increase (Gaut et al. 2015). Likewise, no difference in terms of deleterious variant composition was found between sunflower landraces and elite lines (Renaut and Rieseberg 2015). Hence, while the dominant consensus is that the domestication was accompanied by a genetic cost linked to the combined effects of bottlenecks, limited effective recombination reducing selection efficiency, and deleterious allele surfing by rapid population expansion (Moyers et al. 2018), recent breeding highlights a different pattern. We argue that our results may help to understand this difference because under High DriftHigh Selection intensity, a regime likely prevalent in modern breeding, genetic load is reduced. Moreover, our results may provide useful hints to explain the evolutionary potential of selfing populations located at the range margins. Just like ours, such populations are generally small, display both, inbreeding and reduced standing variation (Pujol and Pannell 2008) and are subjected to environmental and demographic stochasticity.

Conclusion In conclusion, our High Drift-High Selection intensity regime with non-limiting mutation highlights an interesting interplay between drift and selection that promotes the quick fixation of adaptive de novo mutations fueling a significant but stochastic selection response. Interestingly, such selection response is not impeded by the fixation of deleterious mutations so that adaptation in HDHS proceeds with limited genetic load. Our results provide an explanation for patterns highlighted during recent breeding as well as the high colonization ability of small selfing populations located at species range margins. They also call for a better mathematical description of the multilocus adaptive process sustaining the evolution of small populations 
under intense selection.

\section{Acknowledgements}

We are grateful to Adrienne Ressayre, Elodie Marchadier, Aurélie Bourgais, Sophie Jouanne, Nathalie Galic, Cyril Bauland, and Philippe Jamin who contributed over the years to the field experiments; and to Hélène de Préval and Clément Brusq for their contribution to the simulations.

\section{Funding}

This work was supported by two grants (FloSeq and Itemaize) overseen by the French National Research Agency (ANR) as part of the "Investissements d'Avenir" Programme (LabEx BASC; ANR-11-LABX-0034) to C.D. GQE-Le Moulon benefits from the support of Saclay Plant Sciences-SPS (ANR-17-EUR-0007) as well as from the Institut Diversité, Ecolgie et Evolution du Vivant (IDEEV). A.D.-P. was financed by a doctoral contract from the French ministry of Research through the Doctoral School "Sciences du Végétal: du gène à l'écosystème" (ED 567).

\section{Data availability}

The complete R script for performing the simulations as well as the summary data for flowering time (2 DSEs and 20 generations) are available at INRAe dataverse (data.inrae.fr): https:/ /doi.org/10.15454/JQABMJ

\section{Conflicts of Interest}

The authors declare that there is no conflict of interest.

\section{Literature Cited}

Berg, J. J. and G. Coop, 2014 A Population Genetic Signal of Polygenic Adaptation. PLoS Genetics 10: e1004412.

Buckler, E. S., J. B. Holland, P. J. Bradbury, C. B. Acharya, P. J. Brown, et al., 2009 The genetic architecture of maize flowering time. Science 325: 714-718.

Bulmer, M. G., 1971 The Effect of Selection on Genetic Variability. The American Naturalist 105: 201-211.

Burke, M. K., 2012 How does adaptation sweep through the genome? Insights from long-term selection experiments. Proceedings of the Royal Society B: Biological Sciences 279: 50295038.

Burke, M. K., G. Liti, and A. D. Long, 2014 Standing genetic variation drives repeatable experimental evolution in outcrossing populations of saccharomyces cerevisiae. Molecular Biology and Evolution 31: 3228-3239.

Caballero, A., M. A. Toro, and C. Lopez-Fanjul, 1991 The response to artificial selection from new mutations in Drosophila melanogaster. Genetics 128: 89-102.

Camus-Kulandaivelu, L., J. B. Veyrieras, D. Madur, V. Combes, M. Fourmann, et al., 2006 Maize adaptation to temperate climate: Relationship between population structure and polymorphism in the Dwarf8 gene. Genetics 172: 2449-2463.

Carr, R. N. and R. F. Nassar, 1970 Effects of Selection and Drift on the Dynamics of Finite Populations. I. Ultimate Probability of Fixation of a Favorable Allele. Biometrics 26: 41.

Chantepie, S. and L. Chevin, 2020 How does the strength of selection influence genetic correlations? Evolution Letters 4: 468-478.

Charlesworth, B., 1993 Directional selection and the evolution of sex and recombination. Genetical Research 61: 205-224.
Chevin, L. M. and F. Hospital, 2008 Selective sweep at a quantitative trait locus in the presence of background genetic variation. Genetics 180: 1645-1660.

Clark, R. M., S. Tavaré, and J. Doebley, 2005 Estimating a nucleotide substitution rate for maize from polymorphism at a major domestication locus. Molecular Biology and Evolution 22: 2304-2312.

Clayton, G. and A. Robertson, 1955 Mutation and Quantitative Variation. The American Naturalist 89: 151-158.

Crow, J. F. and M. Kimura, 1971 An Introduction to Population Genetics Theory, volume 26. Blackburn Press.

Crow, J. F. and M. Kimura, 1979 Efficiency of truncation selection. Proceedings of the National Academy of Sciences of the United States of America 76: 396-399.

Crow, J. F. and T. Maruyama, 1971 The number of neutral alleles maintained in a finite, geographically structured population. Theoretical Population Biology 2: 437-453.

De Leon, N. and J. G. Coors, 2002 Twenty-four cycles of mass selection for prolificacy in the Golden Glow maize population. Crop Science 42: 325-333.

Desai, M. M. and D. S. Fisher, 2007 Beneficial mutation-selection balance and the effect of linkage on positive selection. Genetics 176: 1759-1798.

Dillmann, C. and J. L. Foulley, 1998 Another look at multiplicative models in quantitative genetics. Genetics Selection Evolution 30: 543-564.

Dudley, J. W. and R. J. Lambert, 2010100 Generations of Selection for Oil and Protein in Corn. In Plant Breeding Reviews, volume 24, pp. 79-110, John Wiley and Sons, Inc., Oxford, UK.

Durand, E., S. Bouchet, P. Bertin, A. Ressayre, P. Jamin, et al., 2012 Flowering time in maize: Linkage and epistasis at a major effect locus. Genetics 190: 1547-1562.

Durand, E., M. I. Tenaillon, X. Raffoux, S. Thépot, M. Falque, et al., 2015 Dearth of polymorphism associated with a sustained response to selection for flowering time in maize. BMC Evolutionary Biology 15: 103.

Durand, E., M. I. Tenaillon, C. Ridel, D. Coubriche, P. Jamin, et al., 2010 Standing variation and new mutations both contribute to a fast response to selection for flowering time in maize inbreds. BMC Evolutionary Biology 10: 2.

Falconer, D. S., 1971 Improvement of litter size in a strain of mice at a selection limit. Genetical Research 17: 215-235.

Fisher, R. A., 1930 The genetical theory of natural selection.. OxfordClarendon Press.

Gaut, B. S., C. M. Díez, and P. L. Morrell, 2015 Genomics and the Contrasting Dynamics of Annual and Perennial Domestication. Trends in Genetics 31: 709-719.

Gerrish, P. J. and R. E. Lenski, 1998 The fate of competing beneficial mutations in an asexual population. Genetica 102-103: 127-144.

Good, B. H., M. J. McDonald, J. E. Barrick, R. E. Lenski, and M. M. Desai, 2017 The dynamics of molecular evolution over 60,000 generations. Nature 551: 45-50.

Good, B. H., I. M. Rouzine, D. J. Balick, O. Hallatschek, and M. M. Desai, 2012 Distribution of fixed beneficial mutations and the rate of adaptation in asexual populations. Proceedings of the National Academy of Sciences of the United States of America 109: 4950-4955.

Haldane, J. B. S., 1927 A Mathematical Theory of Natural and Artificial Selection, Part V: Selection and Mutation. Mathematical Proceedings of the Cambridge Philosophical Society 23: 838-844. 
Hartfield, M., T. Bataillon, and S. Glémin, 2017 The Evolutionary Interplay between Adaptation and Self-Fertilization. Trends in Genetics 33: 420-431.

Hartfield, M. and S. Glémin, 2014 Hitchhiking of deleterious alleles and the cost of adaptation in partially selfing species. Genetics 196: 281-293.

Hermisson, J. and P. S. Pennings, 2017 Soft sweeps and beyond: understanding the patterns and probabilities of selection footprints under rapid adaptation. Methods in Ecology and Evolution 8: 700-716.

Hill, W. G., 1982a Predictions of response to artificial selection from new mutations. Genetical Research 40: 255-278.

Hill, W. G., 1982b Rates of change in quantitative traits from fixation of new mutations. Proceedings of the National Academy of Sciences of the United States of America 79: 142-145.

Hill, W. G. and A. Caballero, 1992 Artificial Selection Experiments. Annual Review of Ecology and Systematics 23: 287310.

Hill, W. G., M. E. Goddard, and P. M. Visscher, 2008 Data and theory point to mainly additive genetic variance for complex traits. PLoS Genetics 4: e1000008.

Hill, W. G. and J. Rasbash, 1986 Models of long-term artificial selection in finite population with recurrent mutation. Genetical Research 48: 125-131.

Hill, W. G. and A. Robertson, 1966 The effect of linkage on limits to artificial selection. Genetical Research 8: 269-294.

Hodgins-Davis, A., D. P. Rice, J. P. Townsend, and J. Novembre, 2015 Gene expression evolves under a house-of-cards model of stabilizing selection. Molecular Biology and Evolution 32: 2130-2140.

Höllinger, I., P. S. Pennings, and J. Hermisson, 2019 Polygenic adaptation: From sweeps to subtle frequency shifts. PLoS Genetics 15: e1008035.

Hospital, F. and C. Chevalet, 1996 Interactions of selection, linkage and drift in the dynamics of polygenic characters. Genetical Research 67: 77-87.

Houle, D., 1989 The Maintenance of Polygenic Variation in Finite Populations. Evolution 43: 1767.

Jiao, Y., P. Peluso, J. Shi, T. Liang, M. C. Stitzer, et al., 2017 Improved maize reference genome with single-molecule technologies. Nature 546: 524-527.

Kamran-Disfani, A. and A. F. Agrawal, 2014 Selfing, adaptation and background selection in finite populations. Journal of Evolutionary Biology 27: 1360-1371.

Kassen, R. and T. Bataillon, 2006 Distribution of fitness effects among beneficial mutations before selection in experimental populations of bacteria. Nature Genetics 38: 484-488.

Kawecki, T. J., R. E. Lenski, D. Ebert, B. Hollis, I. Olivieri, et al., 2012 Experimental evolution. Trends in Ecology and Evolution 27: 547-560.

Keightley, P. D., 1994 The distribution of mutation effects on viability in Drosophila melanogaster. Genetics 138: 1315-1322.

Keightley, P. D., 1996 Metabolic models of selection response. Journal of Theoretical Biology 182: 311-316.

Keightley, P. D., 2010 Mutational Variation and Long-Term Selection Response. In Plant Breeding Reviews, pp. 227-247, John Wiley and Sons, Inc., Oxford, UK.

Kimura, M., 1962 On the probability of fixation of mutant genes in a population. Genetics 47: 713-719.

Kimura, M., 1965 A stochastic model concerning the maintenance of genetic variability in quantitative characters. Proceedings of the National Academy of Sciences of the United
States of America 54: 731-736.

Kimura, M., 1969 The number of heterozygous nucleotide sites maintained in a finite population due to steady flux of mutations. Genetics 61: 893-903.

Kimura, M., 1979 Model of effectively neutral mutations in which selective constraint is incorporated. Proceedings of the National Academy of Sciences 76: 3440-3444.

Kimura, M., 1983 The Neutral Theory of Molecular Evolution, volume 54. Cambridge University Press.

Kingman, J. F. C., 1978 A simple model for the balance between selection and mutation. Journal of Applied Probability 15: 1-12.

Lamkey, K., 1992 Fifty years of recurrent selection in the Iowa stiff stalk synthetic maize population. Maydica 37: 19-28.

Lande, R., 1979 Quantitative Genetic Analysis of Multivariate Evolution, Applied to Brain: Body Size Allometry. Evolution 33: 402.

Lande, R. and S. J. Arnold, 1983 The Measurement of Selection on Correlated Characters. Evolution 37: 1210.

Lillie, M., C. F. Honaker, P. B. Siegel, and Ö. Carlborg, 2019 Bidirectional selection for body weight on standing genetic variation in a chicken model. G3: Genes, Genomes, Genetics 9: 1165-1173.

Lush, J. L., 1943 Animal breeding plans. Animal breeding plans .

Lynch, M., B. Walsh, and Others, 1998 Genetics and analysis of quantitative traits, volume 1. Sinauer Sunderland, MA.

Mackay, T. F., 2010 Mutations and quantitative genetic variation: Lessons from Drosophila. Philosophical Transactions of the Royal Society B: Biological Sciences 365: 1229-1239.

Messer, P. W. and D. A. Petrov, 2013 Population genomics of rapid adaptation by soft selective sweeps. Trends in Ecology and Evolution 28: 659-669.

Moose, S. P., J. W. Dudley, and T. R. Rocheford, 2004 Maize selection passes the century mark: A unique resource for 21st century genomics. Trends in Plant Science 9: 358-364.

Moyers, B. T., P. L. Morrell, and J. K. McKay, 2018 Genetic costs of domestication and improvement. Journal of Heredity 109: 103-116.

Neher, R. A., 2013 Genetic Draft, Selective Interference, and Population Genetics of Rapid Adaptation. Annual Review of Ecology, Evolution, and Systematics 44: 195-215.

Odhiambo, M. O. and W. A. Compton, 1987 Twenty Cycles of Divergent Mass Selection for Seed Size in Corn 1. Crop Science 27: 1113-1116.

Parent, B., O. Turc, Y. Gibon, M. Stitt, and F. Tardieu, 2010 Modelling temperature-compensated physiological rates, based on the co-ordination of responses to temperature of developmental processes. Journal of Experimental Botany 61: 2057-2069.

Piganeau, G. and A. Eyre-Walker, 2003 Estimating the distribution of fitness effects from DNA sequence data: Implications for the molecular clock. Proceedings of the National Academy of Sciences 100: 10335-10340.

Plucain, J., T. Hindre, M. Le Gac, O. Tenaillon, S. Cruveiller, et al., 2014 Epistasis and Allele Specificity in the Emergence of a Stable Polymorphism in Escherichia coli. Science 343: 13661369.

Pujol, B. and J. R. Pannell, 2008 Reduced responses to selection after species range expansion. Science 321: 96.

Rebourg, C., M. Chastanet, B. Gouesnard, C. Welcker, P. Dubreuil, et al., 2003 Maize introduction into Europe: The history reviewed in the light of molecular data. Theoretical and Applied Genetics 106: 895-903. 
Renaut, S. and L. H. Rieseberg, 2015 The accumulation of deleterious mutations as a consequence of domestication and improvement in sunflowers and other compositae crops. Molecular Biology and Evolution 32: 2273-2283.

Roberts, R. C., 1967 The limits to artificial selection for body weight in the mouse: IV. Sources of new genetic variance-irradiation and outcrossing. Genetical Research 9: 8798.

Robertson, A., 1960 A theory of limits in artificial selection. Proceedings of the Royal Society of London. Series B. Biological Sciences 153: 234-249.

Romero Navarro, J. A., M. Willcox, J. Burgueño, C. Romay, K. Swarts, et al., 2017 A study of allelic diversity underlying flowering-time adaptation in maize landraces. Nature Genetics 49: 476-480.

Roze, D., 2016 Background selection in partially selfing populations. Genetics 203: 937-957.

Santiago, E. and A. Caballero, 1995 Effective size of populations under selection. Genetics 139: 1013-1030.

Shaw, F. H., C. J. Geyer, and R. G. Shaw, 2002 A comprehensive model of mutations affecting fitness and inferences for Arabidopsis thaliana. Evolution 56: 453-463.

Silander, O. K., O. Tenaillon, and L. Chao, 2007 Understanding the evolutionary fate of finite populations: The dynamics of mutational effects. PLoS Biology 5: 922-931.

Spor, A., D. J. Kvitek, T. Nidelet, J. Martin, J. Legrand, et al., 2014 Phenotypic and genotypic convergences are influenced by historical contingency and environment in yeast. Evolution 68: 772-790.

Stetter, M. G., K. Thornton, and J. Ross-Ibarra, 2018 Genetic architecture and selective sweeps after polygenic adaptation to distant trait optima. PLoS Genetics 14: 1-24.

Tavaré, S., 1984 Line-of-descent and genealogical processes, and their applications in population genetics models. Theoretical Population Biology 26: 119-164.

Tenaillon, M. I., K. Seddiki, M. Mollion, M. L. Guilloux, E. Marchadier, et al., 2018 Transcriptomic response to divergent selection for flowering times reveals convergence and key players of the underlying gene regulatory network. bioRxiv 7: 461947.

Turelli, M., 1984 Heritable genetic variation via mutationselection balance: Lerch's zeta meets the abdominal bristle. Theoretical Population Biology 25: 138-193.

Walsh, B. and M. Lynch, 2018 Evolution and Selection of Quantitative Traits, volume 1. Oxford University Press.

Weber, K. E., 1990 Increased selection response in larger populations. I. Selection for wing-tip height in Drosophila melanogaster at three population sizes. Genetics 125: 579-84.

Weber, K. E., 1996 Large genetic change at small fitness cost in large populations of Drosophila melanogaster selected for wind tunnel flight: rethinking fitness surfaces. Genetics 144: 205-13.

Weber, K. E. and L. T. Diggins, 1990 Increased selection response in larger populations. II. Selection for ethanol vapor resistance in Drosophila melanogaster at two population sizes. Genetics 125: 585-597.

Wei, M., A. Caballero, and W. G. Hill, 1996 Selection response in finite populations. Genetics 144: 1961-1974.

Wellenreuther, M. and B. Hansson, 2016 Detecting Polygenic Evolution: Problems, Pitfalls, and Promises. Trends in Genetics 32: 155-164.

Zeng, Z. B. and C. C. Cockerham, 1993 Mutation models and quantitative genetic variation. Genetics 133: 729-736. 
bioRxiv preprint doi: https://doi.org/10.1101/2020.12.22.423930; this version posted July 14, 2021. The copyright holder for this preprint (which was not certified by peer review) is the author/funder, who has granted bioRxiv a license to display the preprint in perpetuity. It is made available under aCC-BY-NC-ND 4.0 International license.

\section{Supplementary material}

\section{Saclay DSE's selection scheme}

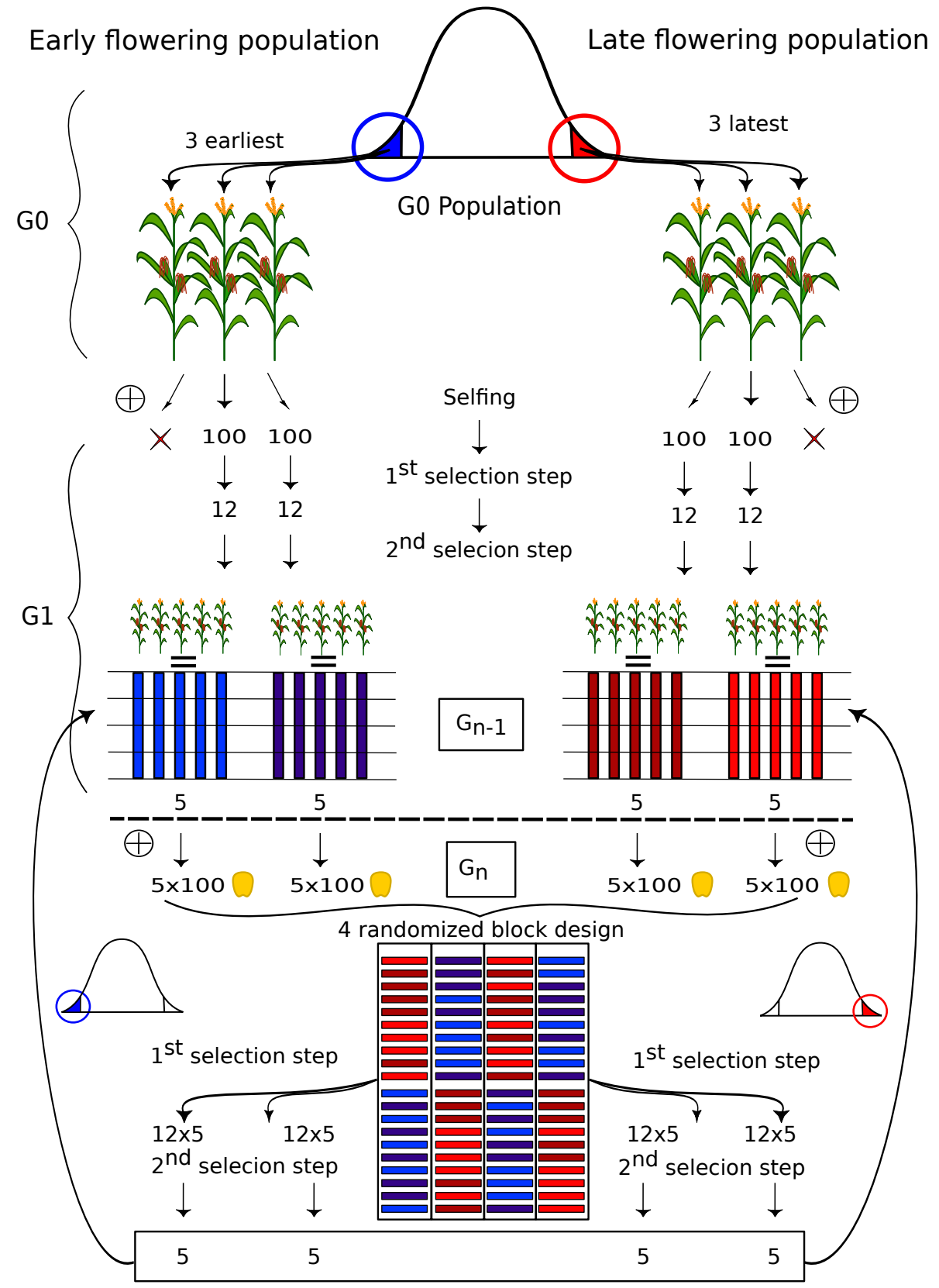

Figure S1 Experimental scheme of Saclay DSEs. For clarity a single scheme is shown but was replicated for the two DSEs. Starting from an inbred $G_{0}$ population with little standing variation ( $<1 \%$ residual heterozygosity (Durand et al. 2015)), the three earliest/ latest flowering individuals represented in blue/ red were chosen based on their offspring phenotypic values as the founders of two families forming the early/ late population. For the subsequent generations, $10(\approx 5$ per family) extreme progenitors were selected in a two step selection scheme among 1000 plants. More specifically, 100 seeds per progenitor were evaluated in a four randomized-block design, i.e. 25 seeds per block in a single row. In a first selection step, the $3 \times 4=12$ earliest/ latest flowering plants among the 100 plants per progenitor were selected in a first step. Then in a second selection step, 10 ( $\approx 5$ per family) individuals were selected within each population based on both flowering time and kernel weight and the additional condition of preserving two progenitors per family from the previous generation. 
(a) ME1

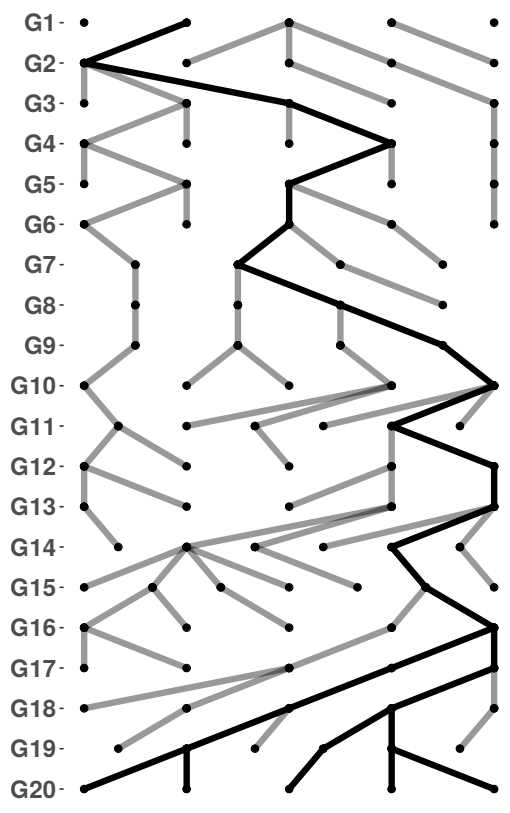

(c) ML1

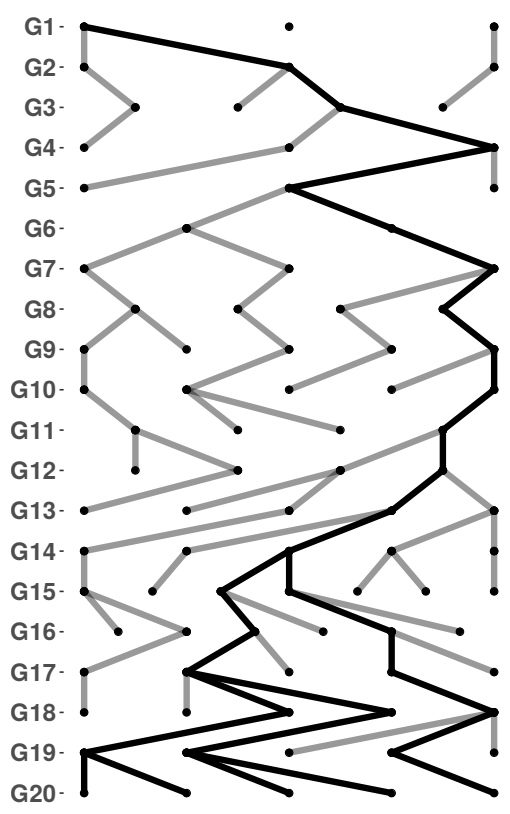

(b) ME2

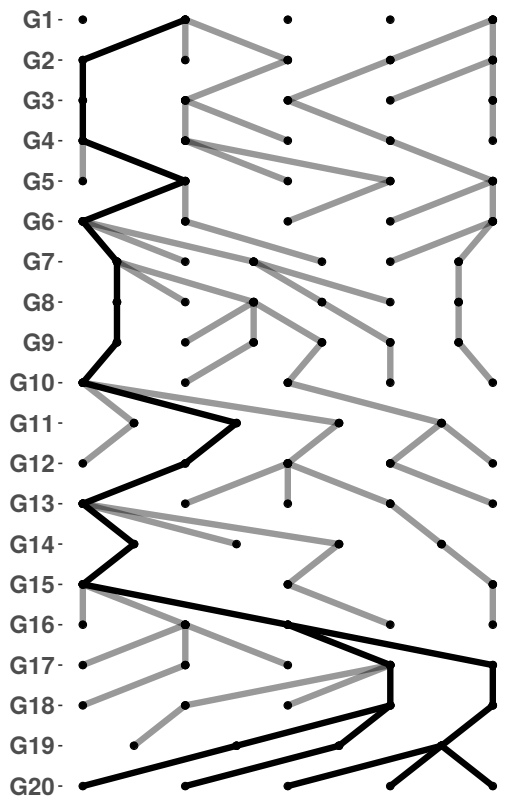

(d) ML2

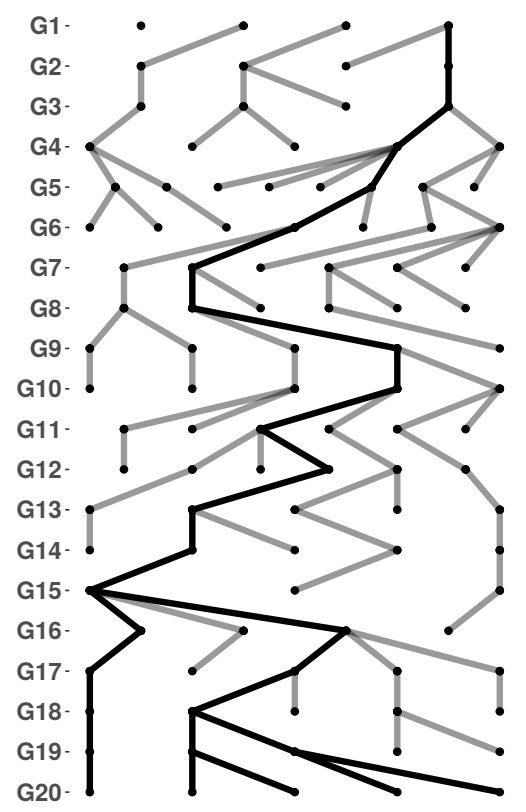

Figure S2 MBS family pedigrees from G1 to $G_{20}$. The two early families ME1 (a) and ME2 (b), and the two late families ML1 (c) and ML2 (d) are presented. Each node corresponds to a progenitor selected at a given generation. Each edge corresponds to a filial relationship between a progenitor and its offspring. Thick black lines indicate the ancestral path of the last generation $\left(G_{20}\right)$. 
(a) FE1

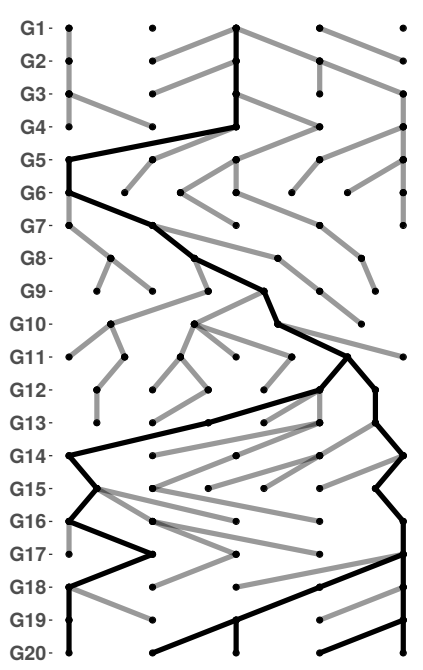

(c) FVL

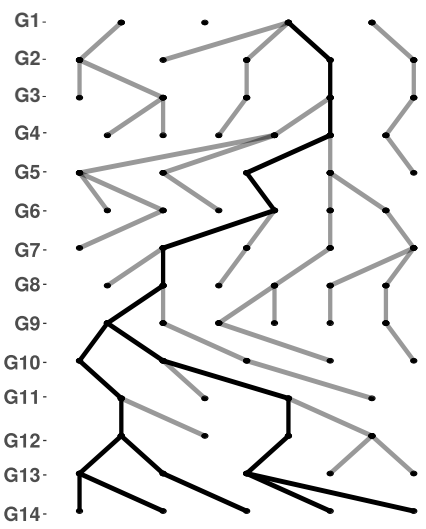

(d) FL2.1

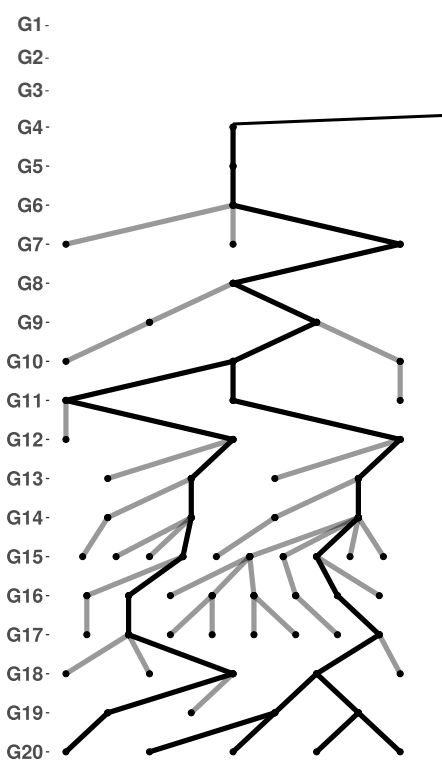

(b) FE2

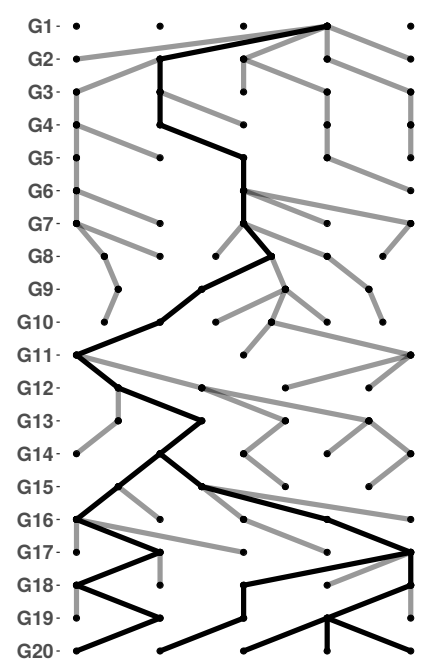

(e) FL2.2

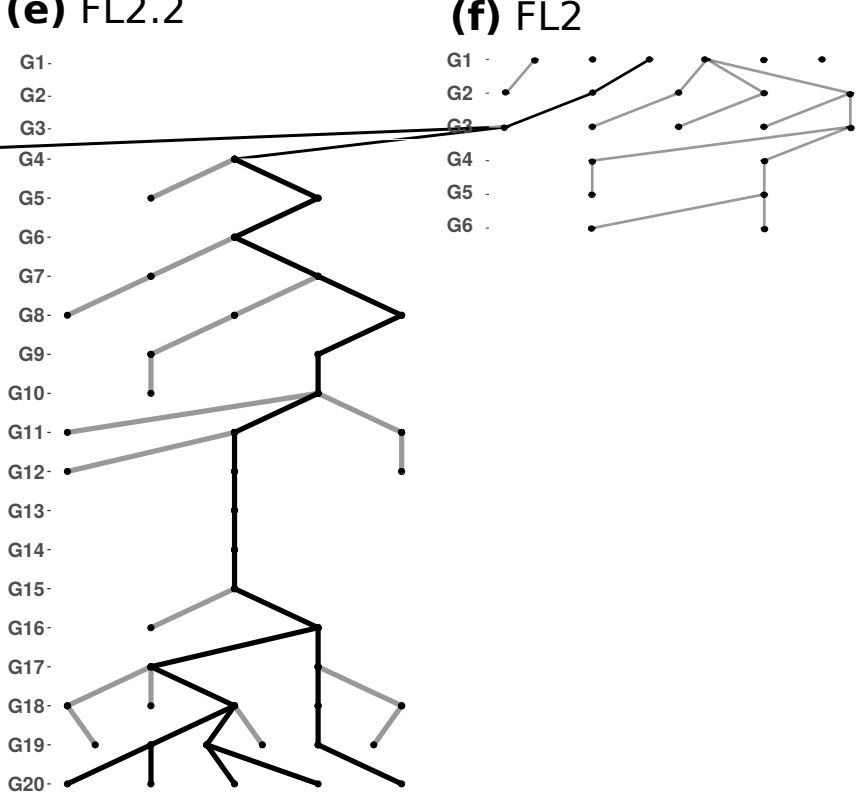

Figure S2 (Continued) F252 family pedigrees from G1 to $G_{20}$. Two early families FE1 (a), FE2 (b) and two late families FVL (c) \& FL2 (f), are represented. FVL (c) could not be maintained after G14 as flowering occurred too late in the season for seed production. Both FL2.1 (d) and FL2.2 (e) were derived from a same individual from FL2 (f) at G3, after FVL was discarded. Each node corresponds to a progenitor selected at a given generation. Each edge corresponds to a filial relationship between a progenitor and its offspring. Thick black lines indicate the ancestral path of the last generation. $\left(G_{20}\right)$ 


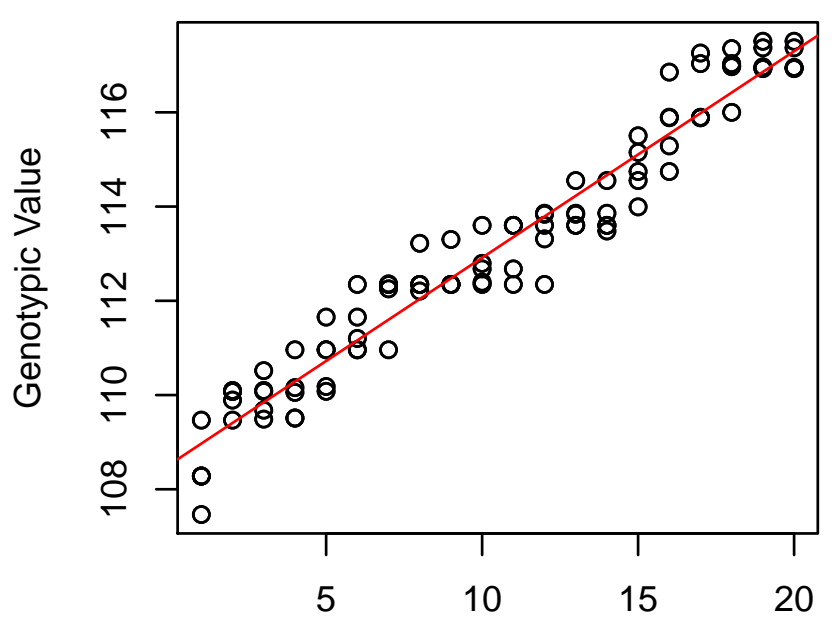

Generation

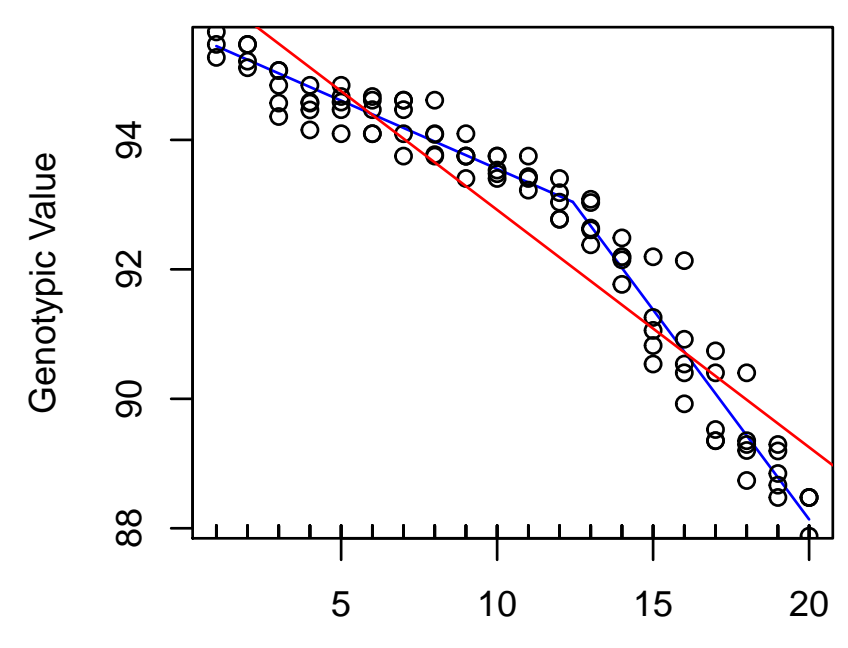

Generation
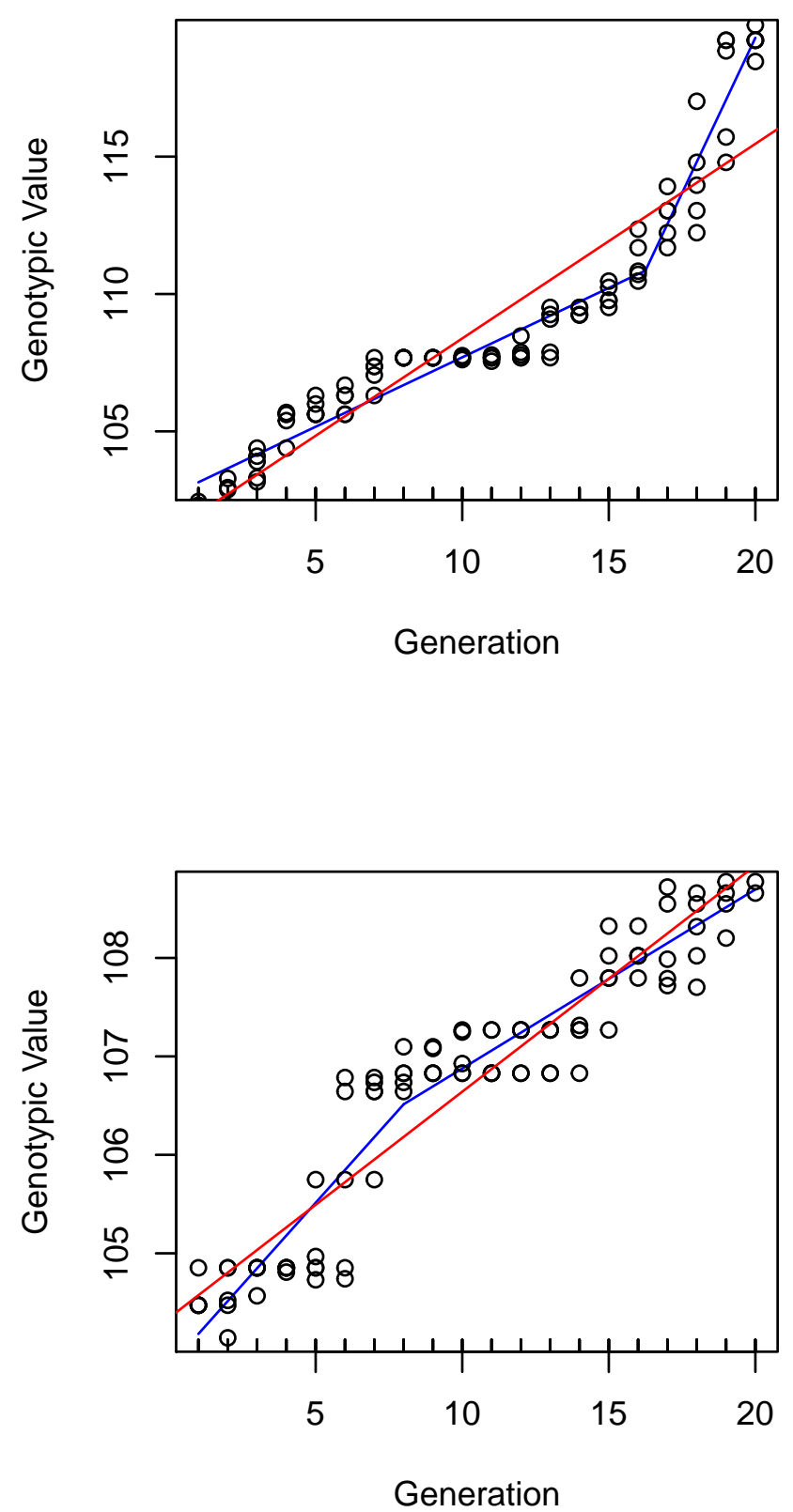

Figure S3 Illustration of simulated non-linear selection response in MBS. Each panel presents the evolution through time (x axis) of the genotypic value (y axis) of the 5 selected individual per family (empty dots). The red lines shows the linear regression of the selected genotypic values through times, while blue lines correspond to the best (AIC criterion) segmented linear model. The top left panel is an example for which a simple linear model fitted best the selection response, while the three others show a diversity of non-linear behaviors. 
bioRxiv preprint doi: https://doi.org/10.1101/2020.12.22.423930; this version posted July 14, 2021. The copyright holder for this preprint (which

was not certified by peer review) is the author/funder, who has granted bioRxiv a license to display the preprint in perpetuity. It is made available under aCC-BY-NC-ND 4.0 International license.

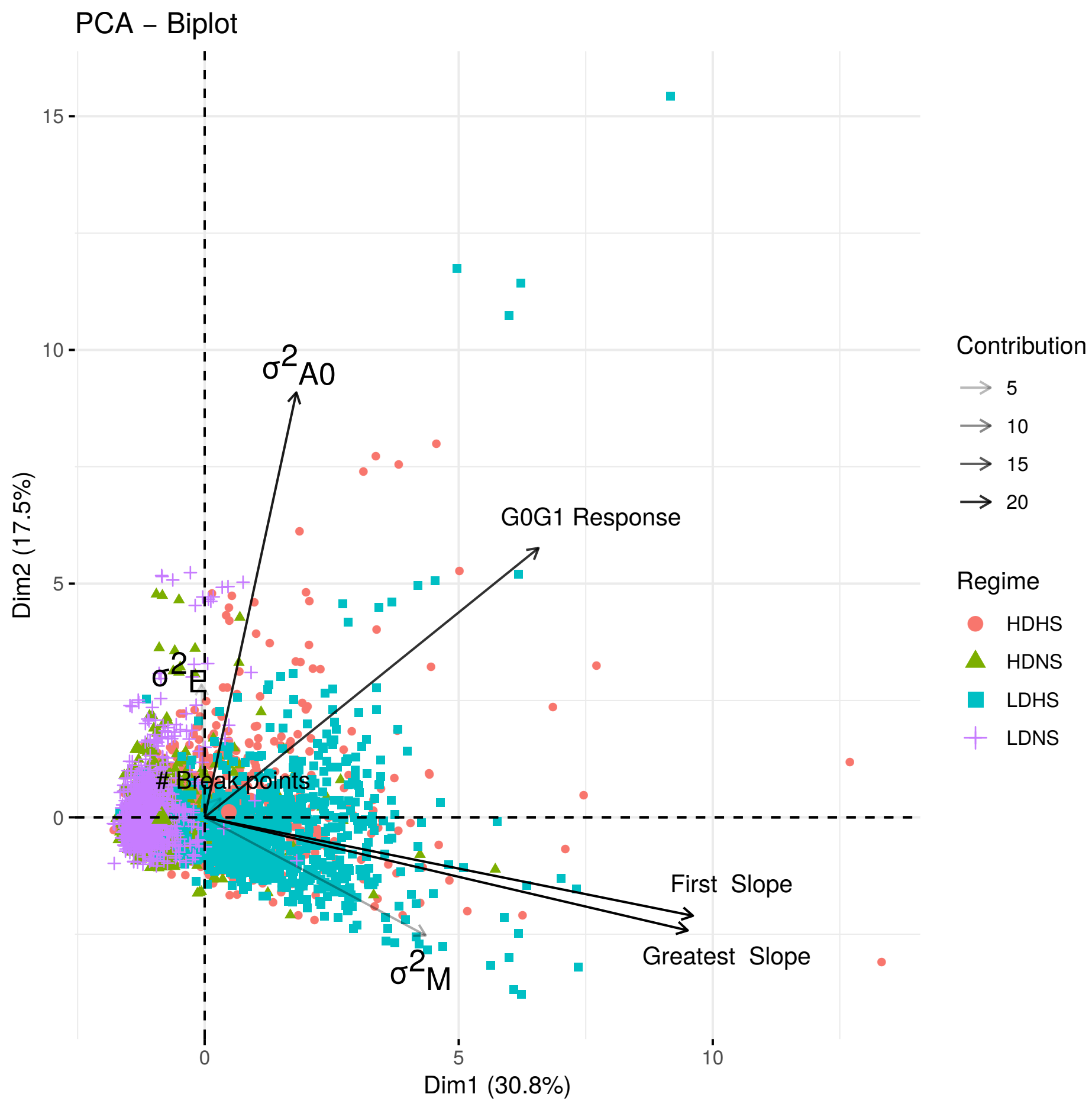

Figure S4 Correlation between model input variables $\left(\sigma_{A 0}^{2}, \sigma_{M}^{2}\right.$ and $\left.\sigma_{E}^{2}\right)$ and output variables $\left(G_{0} G_{1}\right.$ Response, \# Breakpoints, First Slope and Greatest Slope). We obtained the output variables by fitting a segmented linear regression to the selection response from $G_{1}$ to $G_{20}$ in individual. We estimated the number of breakpoints, the corresponding slopes, as well as the first \& greatest slope by AIC maximization. In addition we determined the $G_{0} G_{1}$ response. A Principle Component Analysis was carried out on a subset of 200 independent simulations per regime (HDHS, LDHS, HDNS, LDNS). The darker the arrow representing a variable, the higher the intensity of its correlation to the axes. 
(a) High Drift-High Selection

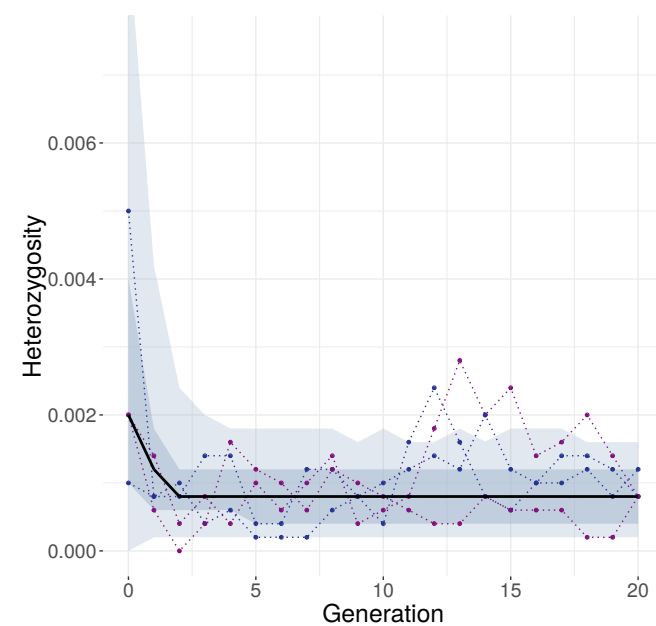

(c) High Drift-No Selection

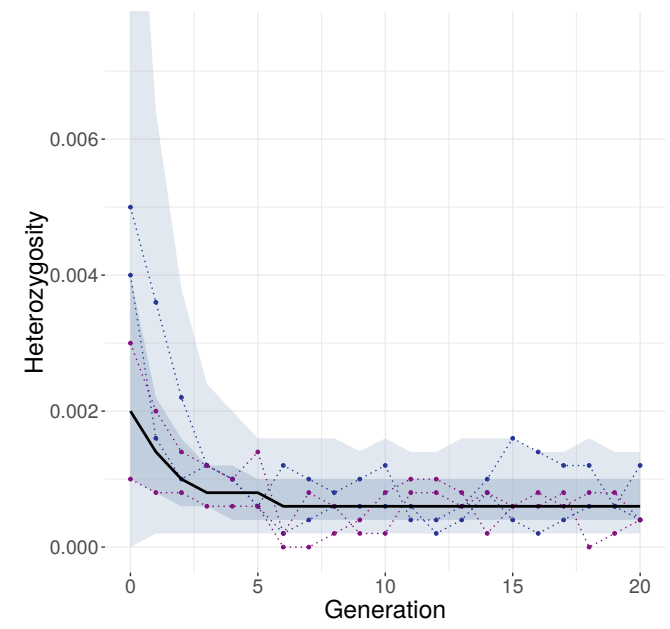

(b) Low Drift-High Selection

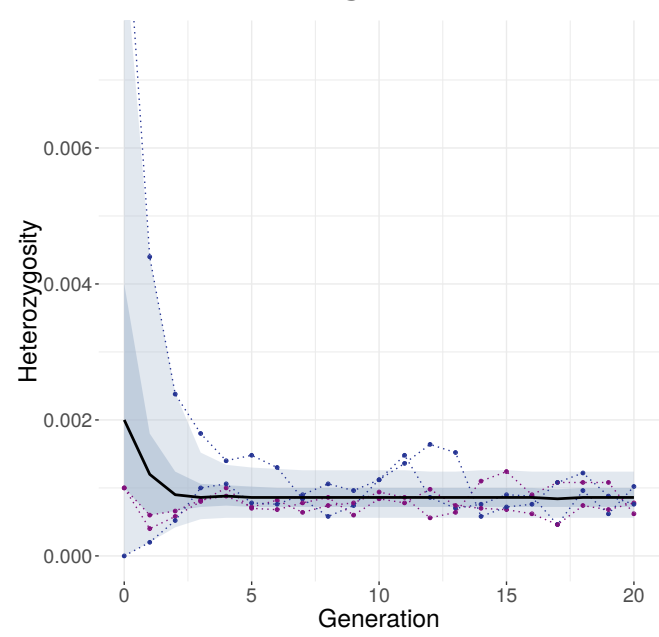

(d) Low Drift-No Selection

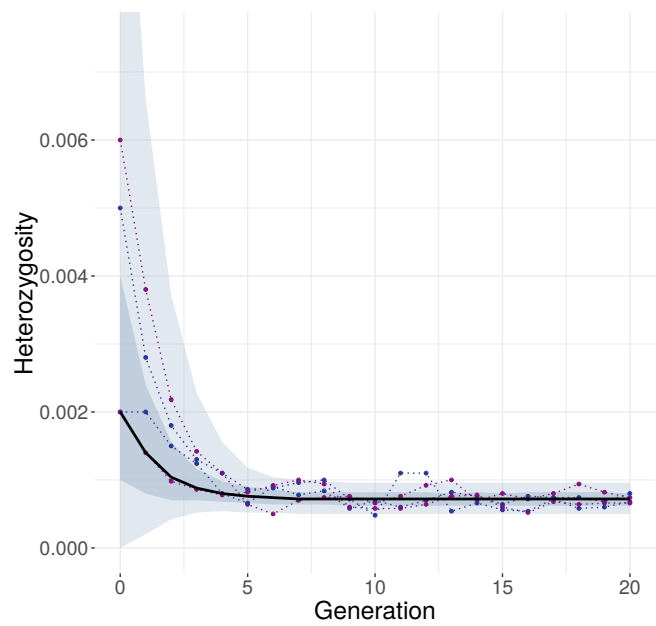

Figure S5 Evolution through time of the per-family mean heterozygosity over all loci, under HDHS (a), LDHS (b), HDNS (c), LDNS (d). The black line represents the median value of the per-family mean heterozygosity. The shaded area corresponds to the $5^{\text {th }}-95^{\text {th }}$ percentile (light blue) and to the $25^{\text {th }}-75^{\text {th }}$ percentile (dark blue). Four randomly chosen simulated families are represented with dotted line. 
bioRxiv preprint doi: https://doi.org/10.1101/2020.12.22.423930; this version posted July 14, 2021. The copyright holder for this preprint (which

was not certified by peer review) is the author/funder, who has granted bioRxiv a license to display the preprint in perpetuity. It is made available under aCC-BY-NC-ND 4.0 International license.

(a) High Drift-High Selection

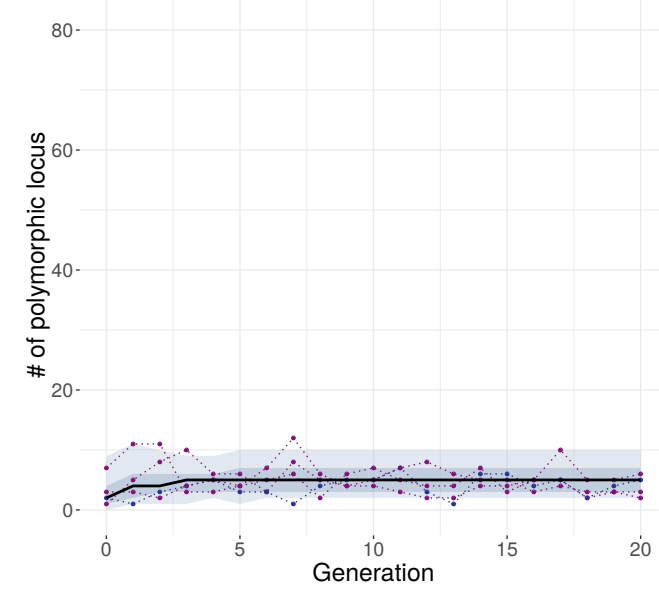

(c) High Drift-No Selection

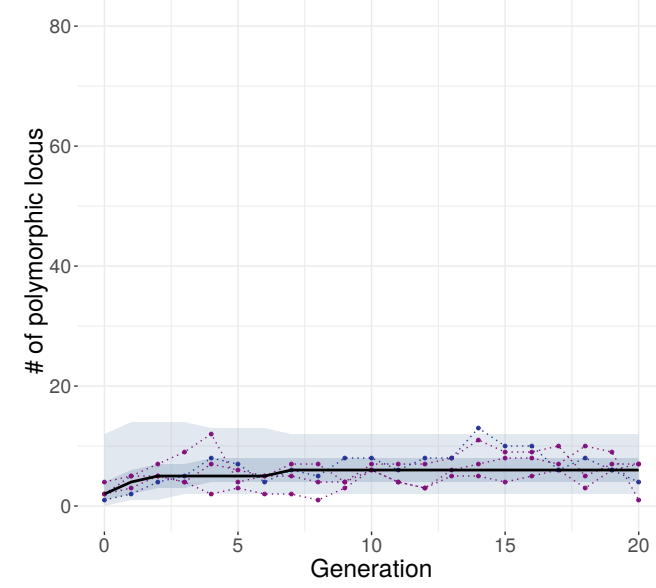

\section{(b) Low Drift-High Selection}

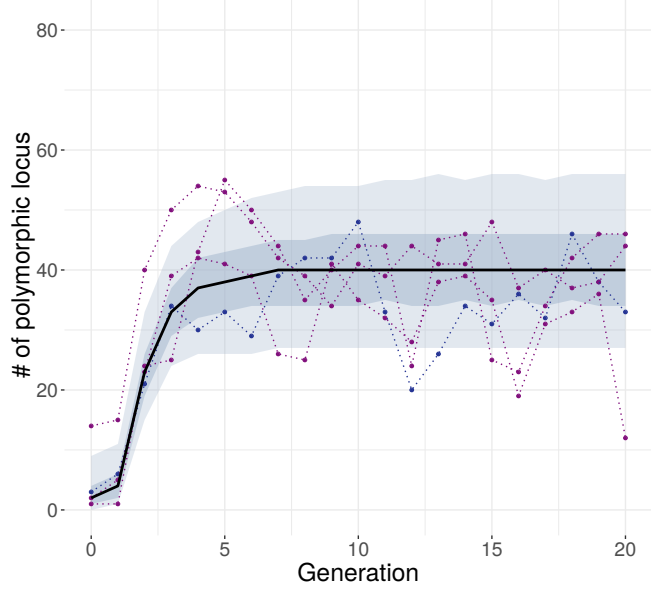

(d) Low Drift-No Selection

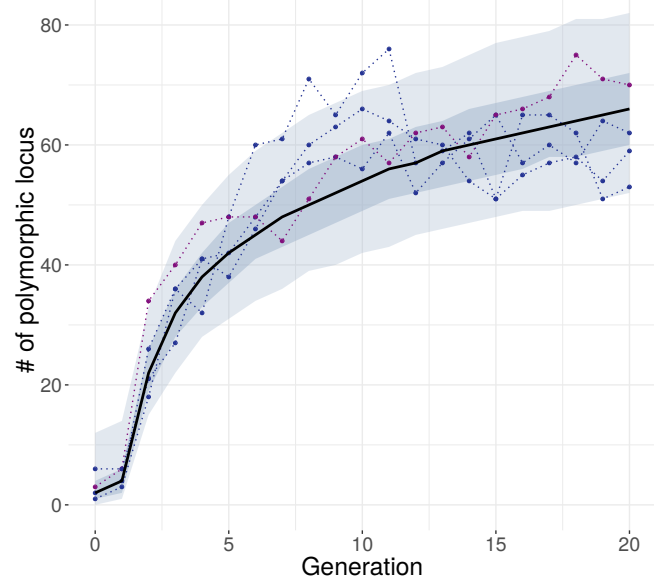

Figure S6 Evolution through time of the per-family mean number of polymorphic loci, under HDHS (a), LDHS (b), HDNS (c), LDNS (d). The black line represents the median value over 2000 simulations. The shaded area corresponds to the $5^{\text {th }}-95^{\text {th }}$ percentile (light blue) and to the $25^{\text {th }}-75^{\text {th }}$ percentile (dark blue). Four randomly chosen simulated families are represented with dotted line. 
bioRxiv preprint doi: https://doi.org/10.1101/2020.12.22.423930; this version posted July 14, 2021. The copyright holder for this preprint (which was not certified by peer review) is the author/funder, who has granted bioRxiv a license to display the preprint in perpetuity. It is made available under aCC-BY-NC-ND 4.0 International license.

\section{(a) High Drift-High Selection}

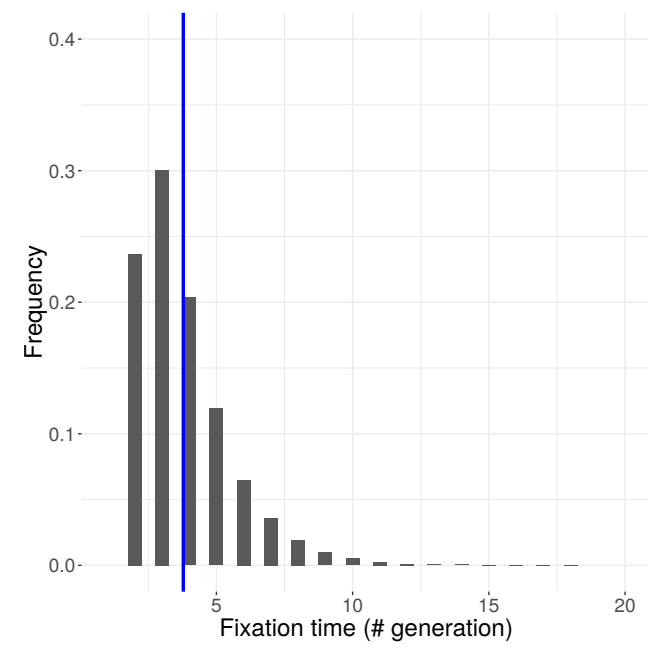

(c) High Drift-No Selection

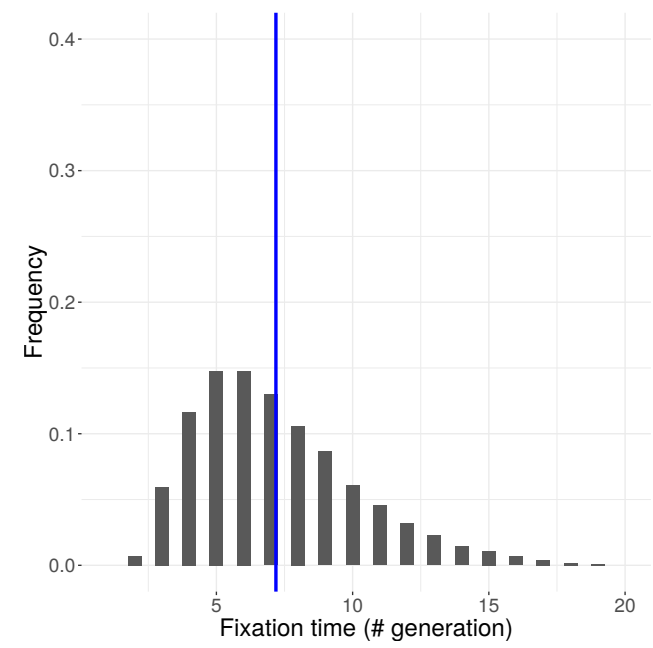

(b) Low Drift-High Selection

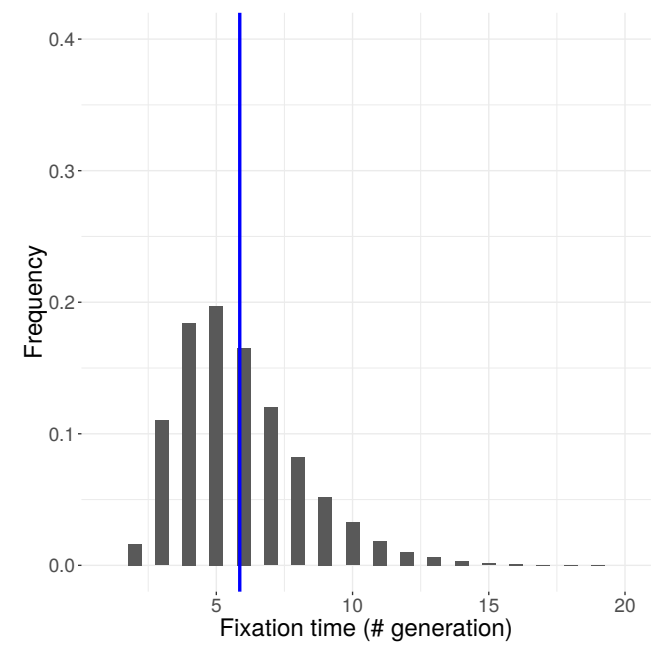

(d) Low Drift-No Selection

Figure S7 Frequency distribution of mutation fixation times over all simulated families under HDHS (a), LDHS (b), HDNS (c), LDNS (d). Note that under LDNS, we obtained very few fixed mutation so that we were unable to draw the corresponding distribution. Blue vertical lines represent the interpolated median. 


\section{(a) High Drift-High Selection}

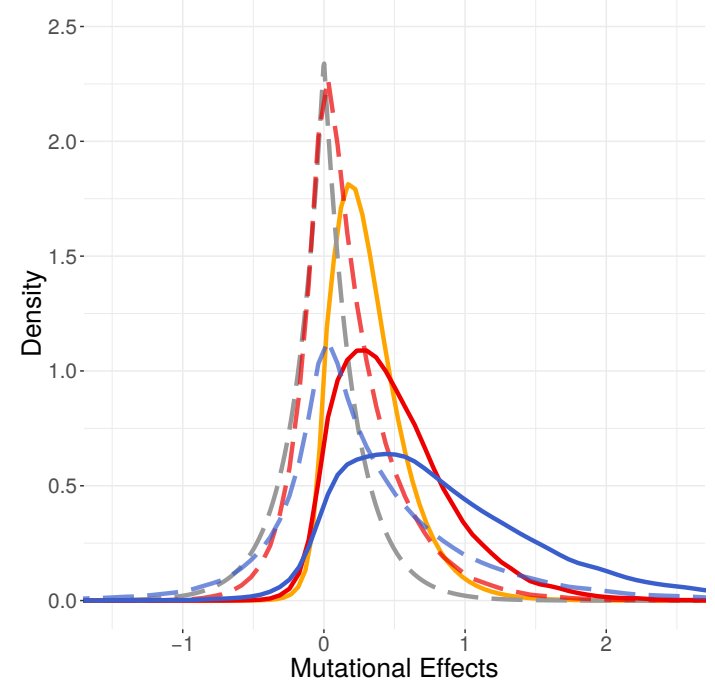

(b) Low Drift-High Selection

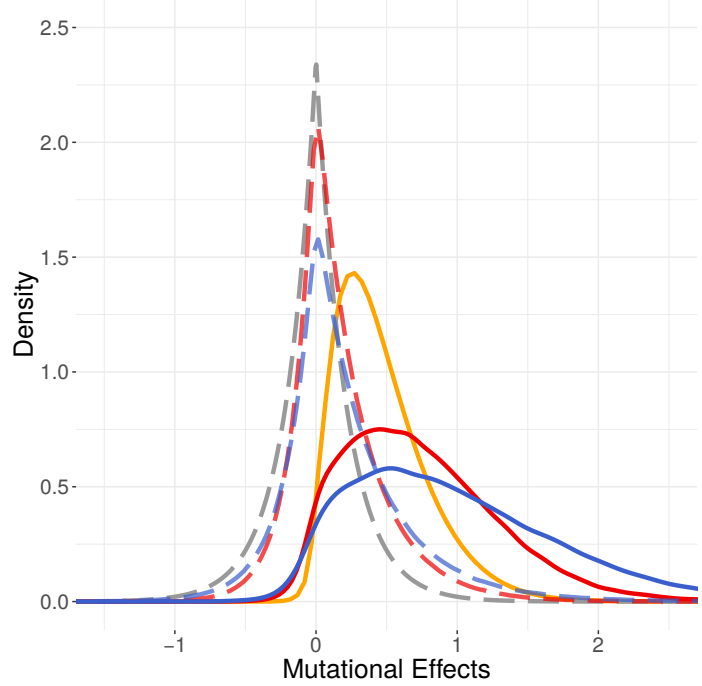

Figure S8 Distribution of mutation effects under HDHS (a), LDHS (b). The dotted lines indicate the distribution of effects (DFE) of incoming de novo mutations considering raw effects in all individuals (grey), in selected individuals (red), and effects normalized by environmental variation in selected individuals (blue). The plain lines indicate DFE of fixed mutations following the same colour code. The golden line represents the expected DFE of fixed mutations according to Eq: 16. 
(a) High Drift-High Selection

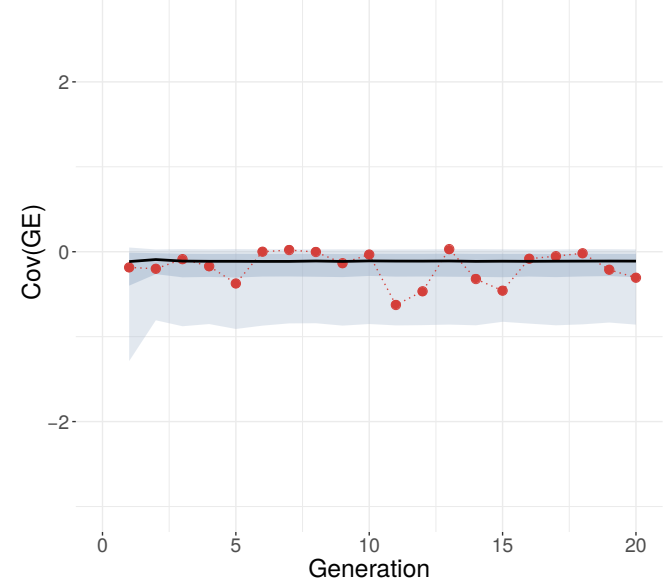

(c) High Drift-No Selection

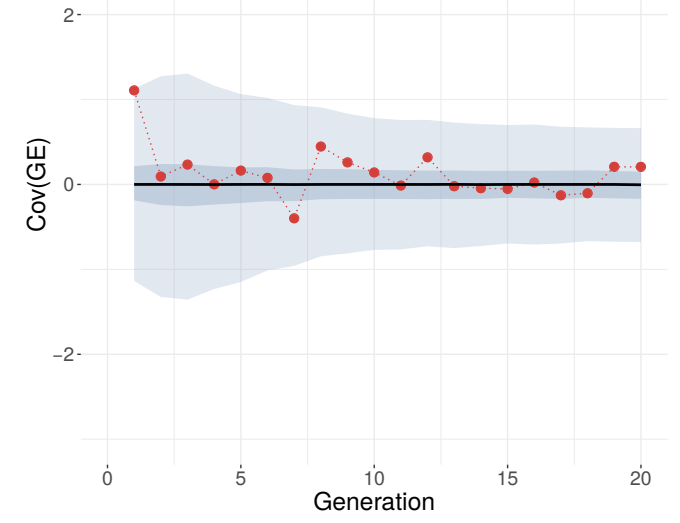

\section{(b) Low Drift-High Selection}

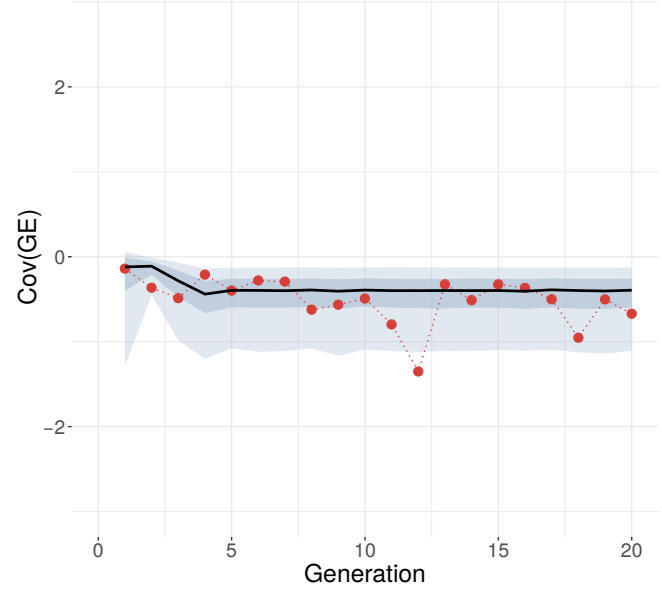

(d) Low Drift-No Selection

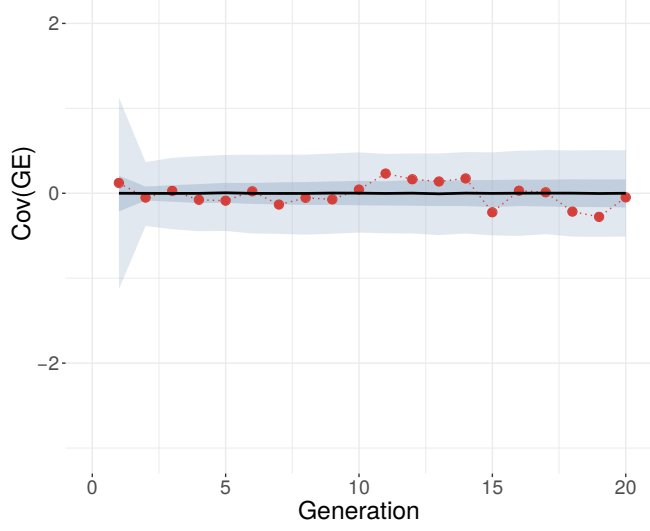

Figure S9 Evolution through time of the per-family covariance between environmental and genotypic values of the selected individuals, under our four simulated regimes. The black line represents the evolution of the median value over 2000 simulations in HDHS (a), LDHS (b), HDNS (c), LDNS (d). The shaded area corresponds to the $5^{\text {th }}-95^{\text {th }}$ percentile (light blue) and to the $25^{\text {th }}-75^{\text {th }}$ percentile (dark blue). One randomly chosen simulated family is represented with red dotted line, to highlight the inter-generation stochasticity. No significant autocorrelation was found. 
bioRxiv preprint doi: https://doi.org/10.1101/2020.12.22.423930; this version posted July 14, 2021. The copyright holder for this preprint (which was not certified by peer review) is the author/funder, who has granted bioRxiv a license to display the preprint in perpetuity. It is made available under aCC-BY-NC-ND 4.0 International license.

\section{Parameter calibration}



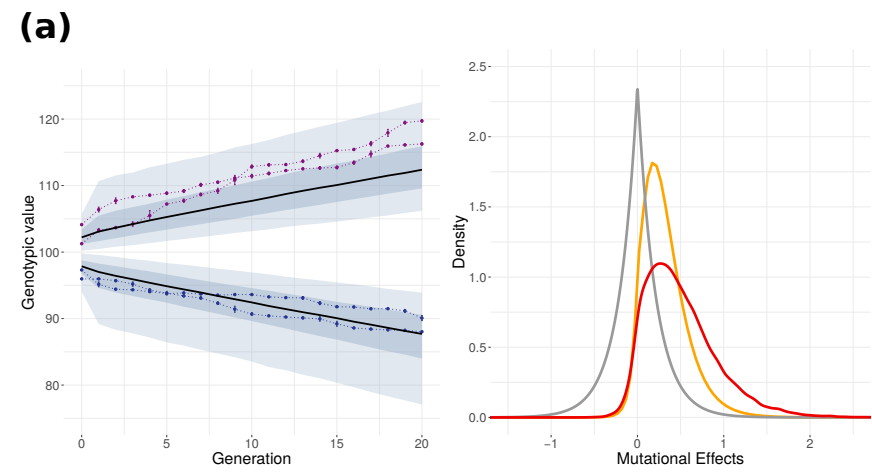

(b)
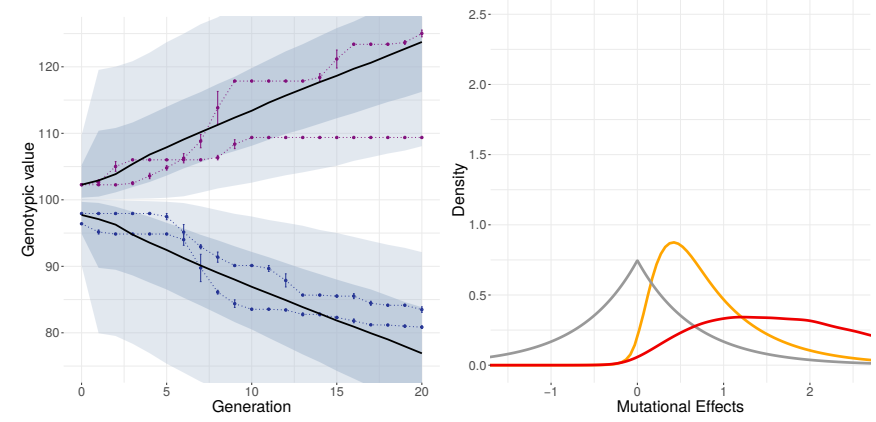

(d)
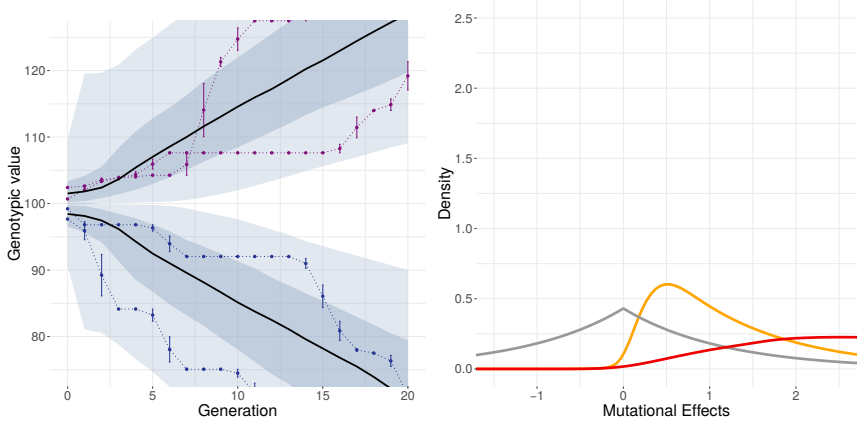

(c)
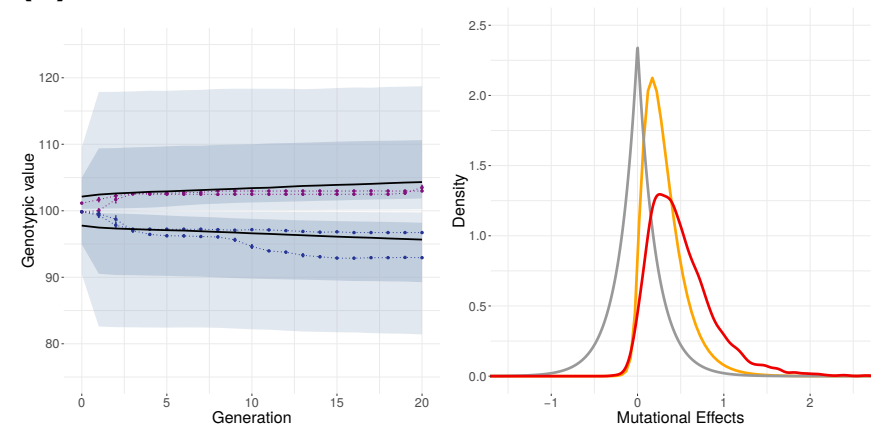

(e)
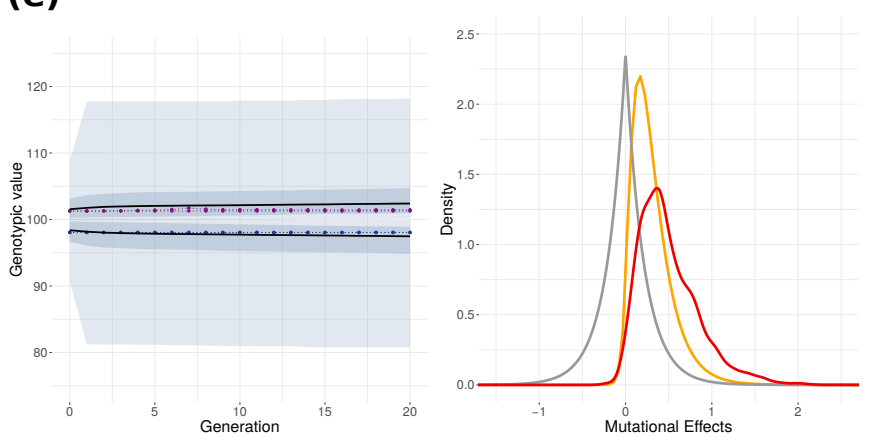

Figure S10 Comparison between simulated HDHS regime under various mutational parameters. Each panel (a) to (e) replicates Fig. 1 on the left and Fig. 4 on the right. The left side of each panel represents the mean genotypic values of the selected progenitors per family (expressed in Days To Flowering, DTF) across generations, violet/blue color identifies the late/ early population. In each population, the black line represents the evolution of the median value over 2000 simulations of the family genotypic mean. The shaded area corresponds to the $5^{\text {th }}-95^{\text {th }}$ percentile (light blue) and to the $25^{\text {th }}-75^{\text {th }}$ percentile (dark blue). In addition, two randomly chosen simulations are shown with dotted lines. The right side of each panel represents the distribution of effects of incoming de novo and fixed mutations under HDHS. Density distributions are shown for all incoming de novo mutational effects in grey reflected exponential distribution -, and fixed mutations over 2000 simulations in red. Theoretical expectations from (Eq: 16) are plotted in gold. Panel (a) corresponds to the main text simulation parameters, so that HDHS: $U=1000 \times 6000 \times 30 \times 10^{-9}=$ $0.18, E\left(\lambda_{M}\right)=4.69, E\left(\sigma_{M}^{2}\right)=0.033$. (b) and (c) correspond to simulations with 100 loci and the same mutation rate per locus $\mu=6000 \times 30 \times 10^{-9}=0.018$, while (d) and (e) correspond to simulations with 1000 loci but a mutation rate per locus $\mu=$ $6000 \times 1 \times 10^{-9}=0.006$. Panels (b) and (d) were obtained with the same total mutational variance $E\left(\sigma_{M}^{2}\right)=0.033$ as HDHS but smaller $\lambda_{M},\left(E\left(\lambda_{M}\right)=1.50\right.$ and $E\left(\lambda_{M}\right)=0.86$ respectively). (c) and (e) were obtained with the same mutational variance per locus as $\operatorname{HDHS}\left(E\left(\lambda_{M}\right)=4.69\right)$ but smaller total mutational variance than HDHS $\left(E\left(\sigma_{M}^{2}\right)=0.0033\right.$ and $E\left(\sigma_{M}^{2}\right)=0.001$ respectively). Overall we have :

(b) $U=100 \times 6000 \times 30 \times 10^{-9}=0.018, E\left(\lambda_{M}\right)=1.50, E\left(\sigma_{M}^{2}\right)=0.033$

(c) $U=100 \times 6000 \times 30 \times 10^{-9}=0.018, E\left(\lambda_{M}\right)=4.69, E\left(\sigma_{M}^{2}\right)=0.0033$

(d) $U=1000 \times 6000 \times 1 \times 10^{-9}=0.006, E\left(\lambda_{M}\right)=0.86, E\left(\sigma_{M}^{2}\right)=0.033$

(e) $U=1000 \times 6000 \times 1 \times 10^{-9}=0.006, E\left(\lambda_{M}\right)=4.69, E\left(\sigma_{M}^{2}\right)=0.001$.

Note that while the distributions of incoming de novo and fixed mutational effects are similar among panels (a), (c), (e), the number of fixed mutations in (c) and (e) is much lower than in (a) accounting for the lack of selection response observed in those panels. 
bioRxiv preprint doi: https://doi.org/10.1101/2020.12.22.423930; this version posted July 14, 2021. The copyright holder for this preprint (which was not certified by peer review) is the author/funder, who has granted bioRxiv a license to display the preprint in perpetuity. It is made available under aCC-BY-NC-ND 4.0 International license.

Negative $\operatorname{Cov}\left(G_{\mid \text {selected }}, E_{\mid \text {selected }}\right)$ schematic

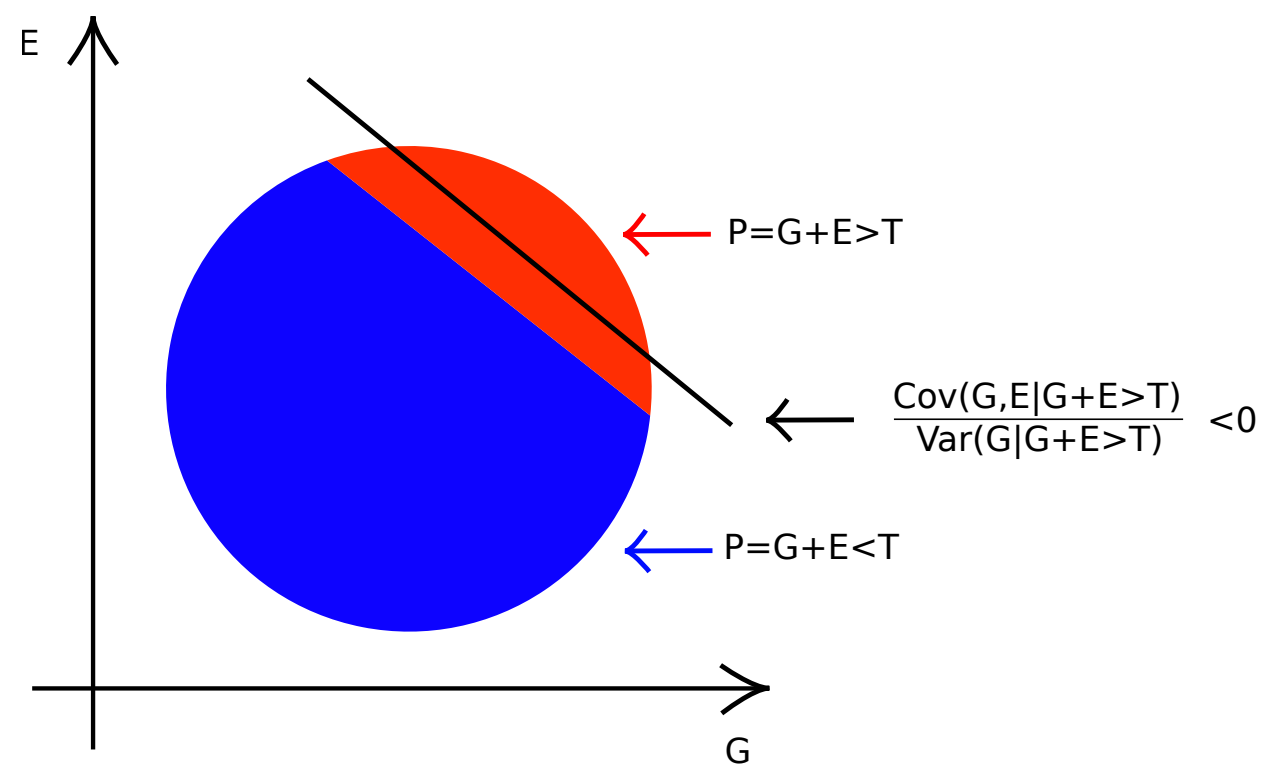

Figure S11 Schematic representation of the impact of selection on $\operatorname{Cov}(G, E)$. For illustration purposes, let $P$ the sum of two independent and identically distributed random variables, $\mathrm{G}$ and $\mathrm{E}$, such that both $\mathrm{G}$ and $\mathrm{E}$ follow a standard normal distribution, i.e. $P=G+E$ with $G \sim \mathcal{N}(0,1)$ and $E \sim \mathcal{N}(0,1)$. The black line represent the regression of $E_{\mid \text {selected }}$ on $G_{\mid \text {selected }}$ with a negative slope $\frac{\operatorname{Cov}\left(G_{\mid \text {selected }}, E_{\mid \text {selected }}\right)}{\operatorname{Var}\left(G_{\mid \text {selected }}\right)} \leq 0$. 
bioRxiv preprint doi: https://doi.org/10.1101/2020.12.22.423930; this version posted July 14, 2021. The copyright holder for this preprint (which was not certified by peer review) is the author/funder, who has granted bioRxiv a license to display the preprint in perpetuity. It is made available under aCC-BY-NC-ND 4.0 International license.

Negative $\operatorname{Cov}\left(G_{\mid \text {selected }}, E_{\mid \text {selected }}\right)$ and its stochasticity

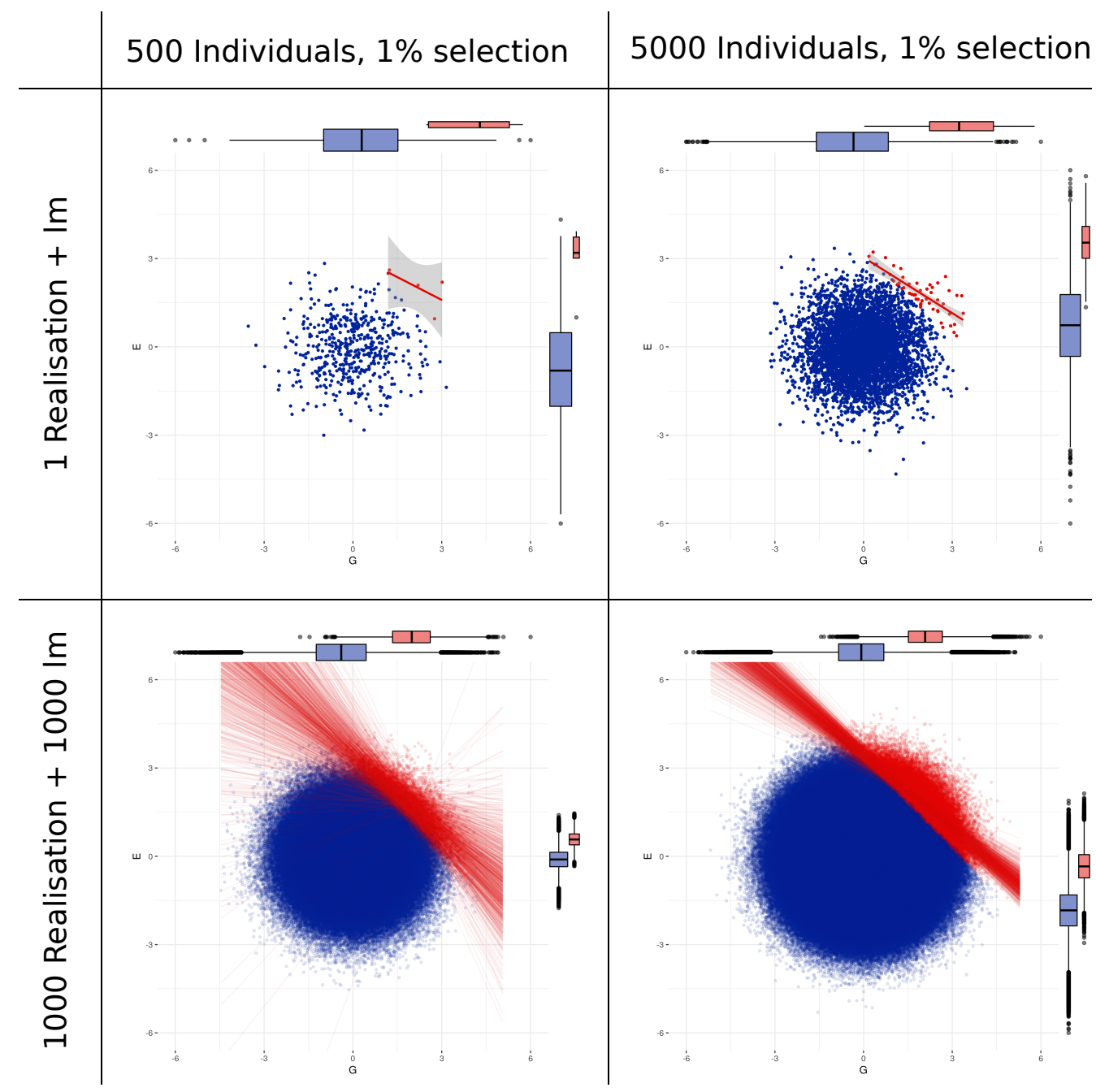

Figure S12 Schematic representation of the impact of selection and drift on $\operatorname{Cov}(G, E)$. Let $P$ the sum of two independent random variables, $\mathrm{G}$ and $\mathrm{E}$, such that both $\mathrm{G}$ and $\mathrm{E}$ follow a standard normal distribution, i.e. $P=G+E$ with $G \sim \mathcal{N}(0,1)$ and $E \sim \mathcal{N}(0,1)$. Let sample 500 individuals from $P$ and plot $E=f(G)$ (right columns), respectively 5000 (left columns) and select (red dots) the best $1 \%$ based on P. The upper row represents one realisation, with the red line corresponding to the regression of $E_{\mid \text {selected }}$ on $G_{\mid \text {selected }}$ with a negative slope $\frac{\operatorname{Cov}\left(G_{\mid \text {selected }}, E_{\mid \text {selected }}\right)}{\operatorname{Var}\left(G_{\text {selected }}\right)} \leq 0$. The lower row represents the realisation of 1000 independent sampling of 500 and 5000 individuals, with the corresponding linear regressions. We observe a lower lesser exploration of possible values (red plus blue area) under low population size and a high stochasticity in the values of $\operatorname{Cov}\left(G_{\mid \text {selected }}, E_{\mid \text {selected }}\right)$ 